Abhandlungen

\title{
Oliver Gapp
}

\section{Die Balanced Scorecard in gesetzlichen Kranken- kassen: Eine empirische Studie zum Stand der Anwendung und deren Ausgestaltung}

Balanced Scorecard; Controlling; Empirische Untersuchung; Gesetzliche Krankenversicherung; Gesundheitswesen; Krankenkassen; Management; Wettbewerb

Das Managementkonzept der Balanced Scorecard (BSC) wird seit wenigen Jahren in Bezug auf dessen Einsatz in gesetzlichen Krankenkassen diskutiert. Bislang fehlen hierzu empirische Untersuchungen. Ziel des vorliegenden Beitrags ist es, in einer empirischen Studie den Bekanntheitsgrad, den Stand der Anwendung sowie den Aufbau und die Ausgestaltung der BSC in gesetzlichen Krankenkassen zu untersuchen. Darüber hinaus werden die Charakteristika der BSC-Krankenkassen und der Einsatz BSC-naher Steuerungsinstrumente dargestellt. Hierzu wurden bei einer schriftlichen Vollerhebung 247 gesetzliche Krankenkassen im Sommer 2006 angeschrieben. Ferner wurden leitfadengestützte Experteninterviews durchgeführt. Die Ergebnisse zeigen, dass sich die BSC als Steuerungsinstrument für gesetzliche Krankenkassen bei entsprechenden Anpassungen gut eignet, insbesondere wegen des Integrationspotenzials qualitativer Erfolgsgrößen. Allerdings wird die BSC in Krankenkassen bislang noch seltener angewandt als in anderen Branchen.

\section{Hintergrund und Zielsetzung}

Die gesetzlichen Krankenkassen sind Non-Profit-Betriebe, die über 70 Millionen Kunden betreuen und Umsätze in der Höhe von $6 \%$ des Bruttoinlandsproduktes erwirtschaften (Böhm et al. 2006, S. 5). Deren Anpassungs- und Restrukturierungsdruck hat in den letzten Jahren deutlich an Dynamik gewonnen. Die anhaltenden Finanzierungsschwierigkeiten, die politischen Diskussionen zur Veränderung geltender Finanzierungs- und Vergütungsmodi sowie weitere rechtliche, medizinische und demographische Faktoren zwingen die gesetzlichen Krankenkassen, neue Lösungen für die gesundheitliche Versorgung der Bevölkerung zu finden (John/Leidl 2004, S. 219; Chow et al. 1998, S. 263). Gleichzeitig müssen sich die Krankenkassen im Wettbewerb untereinander behaupten, welcher innerhalb von zehn Jahren zu einer Reduktion von über 1000 auf weniger als 250 Einzelkassen (2006) geführt hat (Bundesministerium für Gesundheit 2006). 
Während die Regulierung der Sozialversicherung bis in die neunziger Jahre den Krankenkassen nur minimale betriebswirtschaftliche Handlungsspielräume einräumte und sie in erster Linie zu Verwaltungsinstitutionen machte, erlauben neue Rahmenvorgaben heute eine Aktionsbreite, die unternehmerisches Handeln zu einer wichtigen Anforderung in diesem Bereich macht. ${ }^{1}$ Die Rahmenvorgaben betreffen insbesondere die erwähnte Öffnung des Wettbewerbs um Mitglieder wie auch die Einführung verschiedener Gestaltungsoptionen auf der Leistungs- und Versicherungsseite (z. B. Integrierte Versorgung, Disease Management Programme, Gewährung von Selbstbehalten) durch das GKV ${ }^{2}$ Modernisierungsgesetz (2003) und dem GKV-Wettbewerbsstärkungsgesetz (2007). Hinzu kommt die Einführung des Gesundheitsfonds und des morbiditätsorientierten Risikostrukturausgleichs Anfang 2009, was zu großen finanziellen Unsicherheiten für die Krankenkassen führt (Hörbst 2007).

Mit den genannten Herausforderungen geht eine zunehmende Komplexität der Führungsund Leistungssysteme der Krankenkassen einher (Puke 1998, S. 209). Viele gesetzliche Krankenkassen haben jedoch noch keine lange Tradition im Umgang mit betriebswirtschaftlichen Steuerungsinstrumenten (Hofmann 1999, S. 1). Daher ist ein hoher Bedarf an geeigneten Steuerungskonzeptionen und Implementierungswissen bei den gesetzlichen Krankenkassen zu konstatieren (ähnlich Scherer 2002, S. 5).

Ein Managementkonzept, das ursprünglich für die Privatwirtschaft entwickelt wurde und inzwischen auch im Gesundheitswesen diskutiert wird, ist die Balanced Scorecard (BSC) (Kaplan 2001; Kumar et al. 2005). Die BSC operationalisiert mit Hilfe eines Kennzahlensystems die Vision und Strategie eines Unternehmens und passt sie durch regelmäßige Kontrollen den wechselnden Anforderungen an (Kaplan/Norton 1993, S. 135 ff.). Der üblichen Dominanz einer rein finanziellen Betrachtungsweise wird durch das Hinzuziehen nicht-finanzieller Dimensionen entgegengewirkt. Damit greift die BSC Schwachstellen traditioneller Managementsysteme auf, die zumeist Defizite wie eine fehlende Verknüpfung von Strategie und Zielvorgaben oder ein fehlendes strategisches Feedback aufweisen und nicht-finanzielle Parameter nur unzureichend berücksichtigen (Kaplan/Norton 1992, S. 71 ff.). Gerade aus diesen Gründen scheint der Einsatz der BSC in gesetzlichen Krankenkassen besonders interessant. So sind den Krankenkassen als Körperschaften des öffentlichen Rechts im Gegensatz zu privatwirtschaftlich organisierten Unternehmen durch ihre Einbindung in den gesetzlichen Rahmen bestimmte allgemeine Wertvorstellungen sowie der Unternehmenszweck vorgegeben (Haenecke 2001, S. 211). Es steht nicht das Gewinnstreben im Vordergrund, sondern eine Krankenkasse hat die Aufgabe, ausreichende und zweckmäßige Leistungen unter Beachtung des Wirtschaftlichkeitsgebots bereitzustellen ( $\S 1,2,12$ SGB V). Neben den durch den Gesetzgeber definierten Zielen besteht für jede Krankenkasse ein Existenzerhaltungsziel, dessen Realisation eine Voraussetzung zur langfristigen Leistungserbringung ist (Schlösser/

1 Unverkennbar sind inzwischen die Bemühungen der gesetzlichen Krankenkassen zur effizienteren Gestaltung interner Prozesse, zur Ausgliederung bestimmter Aufgabengebiete, zur Kundengewinnung oder zum Aufbau neuer Strukturen (z. B. Internetgeschäftsstellen) (Heinzen 2002, S. 49).

2 Gesetzliche Krankenversicherung. 
Schreyögg 2005, S. 323 f.). Die mehrdimensionale Zielstruktur gesetzlicher Krankenkassen erfordert daher ein Kennzahlensystem, das zur Steuerung der Krankenkassen im Hinblick auf deren Zielerreichung finanzielle und insbesondere nicht-finanzielle Erfolgsgrößen integriert. Weiterhin ist das Vorhandensein von Strategien und damit auch deren Umsetzung in gesetzlichen Krankenkassen bislang kaum gegeben. Argumentiert wird dabei häufig mit einer mangelnden Handlungsfreiheit aufgrund der gesetzlichen Vorgaben, die der Entwicklung und vor allem der Umsetzung von Strategien entgegenstehen (Wesenauer/Bencic 2006, S. 11). Eine BSC-Einführung bietet den Krankenkassen daher die Möglichkeit, den gesamten Strategieprozess aufzunehmen und entsprechend des BSC-Konzepts zu operationalisieren (was der originäre Zweck der BSC ist). Schließlich belegen Berichte/Interviews aus der Praxis, dass die BSC in Krankenkassen eingesetzt wird und damit auch grundsätzlich anwendbar ist. ${ }^{3}$

In der Unternehmenspraxis ist das Konzept der BSC inzwischen breit akzeptiert. Die Anwendungsbereiche reichen von Industriebetrieben (z. B. Fink/Grundler 1998; Fließ et al. 2006) über staatliche Institutionen (z. B. Kaplan/Norton 1997, S. 167 ff.) bis hin zu privaten und öffentlichen Institutionen im Gesundheitswesen - insbesondere Krankenhäusern - (z. B. Borges/Schmidt 2002; Dick et al. 2002). Gleichzeitig wurde das Konzept der BSC in den vergangenen Jahren kontrovers diskutiert. Kritiker werfen dem BSCKonzept vor, eher ein Modeprodukt als ein wirklich neuer Managementansatz zu sein (Weber 1999, S. 362). Andere wiederum betonen den durchaus innovativen Charakter einzelner Bestandteile der BSC (Speckbacher/Bischof 2000, S. 796).

In der Literatur liegen eine Reihe publizierter Erfahrungen zur BSC vor, allerdings basieren diese weitgehend auf Untersuchungen erwerbswirtschaftlicher Unternehmen. ${ }^{4}$ Die gesammelten Erfahrungen aus anderen Bereichen sind aber aufgrund der spezifischen Rahmenbedingungen und des Non-Profit-Charakters von gesetzlichen Krankenkassen nicht ohne Weiteres zu übertragen. ${ }^{5}$

Der Übertragung der BSC auf gesetzliche Krankenversicherungen widmen sich bislang nur wenige Publikationen. ${ }^{6}$ Dabei wird das BSC-Grundkonzept mit den vier ,klassischen“" Perspektiven und deren Hierarchie weitgehend übernommen. Dies steht im Widerspruch zu anderen Autorenaussagen zur BSC-Anwendung in öffentlichen Verwaltungen und dem Gesundheitswesen, wo die Dominanz der Finanzperspektive meist nicht akzeptiert wird und die Perspektiven entsprechend den Rahmenbedingungen erweitert oder angepasst werden (Horváth \& Partners 2004, S. 442; Rimar 2000, S. 1186 f.). Eine inhaltlich stärkere Anpassung an die Krankenkassengegebenheiten findet bei der Formu-

Wesenauer 2006; Klusen/Horvath 2005; Blaudszun/Pielniok 2002.

Horváth 1999; Horváth/Gaiser 2000; Ahn 2003; Speckbacher et al. 2003.

Nahe liegend wäre zunächst, Erfahrungen aus der Versicherungsbranche zu übertragen. Ein Blick in die Literatur zeigt jedoch, dass aufgrund des privatwirtschaftlichen Charakters von Versicherungsunternehmen und des Non-Profit-Charakters von gesetzlichen Krankenkassen eine inhaltliche Nähe nur bedingt besteht (z. B. Mayer/ Ahr 2000; Romeike 2003).

6 Demmler 2002; Wesenauer 2002; Schlösser/Schreyögg 2005; Moos/Brüggemann 2006; Wesenauer 2006; Gapp 2007. Die internationalen Beiträge zur BSC in Krankenversicherungen beziehen sich nicht auf die betriebswirtschaftliche, sondern vorwiegend auf die Versorgungs- bzw. Systemebene in den jeweiligen Gesundheitssystemen, weshalb sie an dieser Stelle keine weitere Erwähnung finden (Sahney 1998; Inamdar et al. 2002; Radnor/Lovell 2003). 
lierung der strategischen Ziele bzw. Kennzahlen statt (Schlösser/Schreyögg 2005, S. 341; Wesenauer 2006, S. 28). Eine erste empirische Untersuchung beschäftigt sich mit dem Umsetzungsprozess der BSC in Krankenkassen, jedoch weniger mit deren inhaltlichen Ausgestaltung (Gapp 2007, S. 281 ff.).

Um die weitere Entwicklung der BSC in gesetzlichen Krankenkassen zielgerichtet zu gestalten, sind empirische Studien zum Einsatz der BSC in gesetzlichen Krankenkassen notwendig, was die generelle Zielsetzung des vorliegenden Beitrags ist.

\section{Forschungsfragestellungen}

Im Folgenden werden die der empirischen Untersuchung zugrunde liegenden Forschungsfragen in theoretischer bzw. sachlogischer Weise hergeleitet. Da die vorliegende Forschungsarbeit einen mitunter stark explorativen Charakter besitzt, werden nicht für jeden Teilaspekt der Untersuchung Forschungshypothesen aufgestellt.

Die BSC besitzt inzwischen einen hohen Verbreitungsgrad. Eine Übersichtsarbeit von Bach zu 26 deutschsprachigen empirischen Studien verschiedener Branchen kommt zu dem Ergebnis, dass etwa die Hälfte der befragten Unternehmen die BSC einsetzen (Bach 2006, S. 298). Nach einer Studie von Ahn und Reuter verwendeten 2005 im deutschen Krankenhausbereich rund $12 \%$ der Krankenhäuser die BSC und $22 \%$ befanden sich im Entwicklungsprozess (Ahn/Reuter 2005, S. 614). Für die gesetzlichen Krankenkassen liegen keinerlei solche Daten vor, weshalb Gegenstand der ersten Forschungsfragestellung der Bekanntheitsgrad und die Verbreitung der BSC in gesetzlichen Krankenkassen ist. Bezüglich letzterem ist anzunehmen, dass die Diffusion der BSC in Krankenkassen deutlich unter der von Bach ermittelten Quote, aber auch etwas unter derjenigen von Ahn und Reuter aus dem Krankenhausbereich liegt, da wettbewerbliche Strukturen im Krankenkassenmarkt noch nicht allzu lange vorherrschen und noch keine lange Tradition im Umgang mit betriebswirtschaftlichen Steuerungsinstrumenten besteht.

Nach Ermittlung der Krankenkassen mit BSC-Anwendung stellt sich die Frage, ob diese Kassen bestimmte Eigenschaften gegenüber Kassen ohne BSC-Anwendung aufweisen und ob die BSC einen positiven Einfluss auf die Entwicklung der Versichertenzahlen hat (zweite Forschungsfragestellung). Als ein Charakteristikum dient hierzu die Größe der Krankenkassen, gemessen an deren Versichertenzahl. Die Bandbreite der Versichertenzahlen reicht von wenigen tausend Versicherten kleiner Betriebskrankenkassen bis hin zu über sieben Millionen Versicherten der Barmer. Aufgrund der größeren finanziellen und personellen Ressourcen mitgliedsstarker Krankenkassen ist anzunehmen, dass diese auch verstärkt mit Managementmethoden wie der BSC arbeiten. Zudem erfordern größere Organisationen einen höheren Koordinationsaufwand. Studien aus der Literatur bestätigen diese Hypothese, wonach die Unternehmensgröße als Erklärung für einen verstärkten BSC-Einsatz dient (Bach 2006, S. 298). 
Ein weiterer Differenzierungsparameter der Krankenkassen ist deren Kassenart. Die Krankenkassen sind in sieben Kassenarten unterteilt. ${ }^{7}$ Aufgrund ihrer historischen Prägung weisen sie verschiedene Eigenschaften und auch heute noch teils sehr unterschiedliche Versichertenstrukturen auf (Haenecke 2001, S. 62 ff.). So hatten die 1883 eingeführten Ortskrankenkassen die Aufgabe, ein flächendeckendes Versorgungsangebot zu gewährleisten. Zudem waren sie bis zum Inkrafttreten des Gesundheitsstrukturgesetzes die Auffang- oder Basiskassen (Cassel 1993, S. 19). Der Versichertenkreis der Betriebskrankenkassen war hingegen traditionell auf die Mitarbeiter des Trägerunternehmens und ihre Angehörige beschränkt. Trotz der genannten Unterschiede lassen sich weder sachlogisch noch aus der Literatur Anhaltspunkte dazu finden, dass eine bestimmte Kassenart innovativer im Einsatz von Managementinstrumenten ist. Insofern ist davon auszugehen, dass sich die Kassenarten bezüglich ihrem Anteil an Krankenkassen mit BSC-Einsatz nicht systematisch unterscheiden.

Die Entwicklung der Versichertenzahlen ist ein existenzieller Parameter für die Krankenkassen (Haenecke 2001, S. 168 ff.). Die Versichertenzahlentwicklung könnte im Sinne eines Leistungsparameters der Krankenkassen interpretiert werden. Umfragen zeigen, dass Unternehmen mit BSC-Anwendung sich - in Bezug auf Jahresüberschuss und Umsatzwachstum - zumindest erfolgreicher fühlen als ihre Wettbewerber (Horváth \& Partners 2005, S. 7). Welchen unmittelbaren Anteil die Verwendung der BSC am Unternehmenserfolg hatte konnte dabei zwar nicht abschließend geklärt werden, dennoch lässt sich die Hypothese aufstellen, dass Krankenkassen mit BSC-Einsatz eine im Durchschnitt günstigere Entwicklung der Versichertenzahlen aufweisen als diejenigen ohne.

Im Rahmen der dritten Forschungsfragestellung wird untersucht, ob die Krankenkassen ohne BSC-Einsatz weniger mit (BSC-nahen) Steuerungsinstrumenten arbeiten als diejenigen mit. Eine zentrale Voraussetzung der BSC-Einführung ist das Vorhandensein einer Unternehmensstrategie (Horváth \& Partners 2004, S. 81 ff.). Wie eingangs aber bereits erwähnt, besteht in Krankenkassen ein erhebliches Defizit im Aufstellen von Strategien. Insofern müssten in BSC-Krankenkassen deutlich häufiger Strategien formuliert sein als in Krankenkassen ohne BSC-Einsatz.

Seit einigen Jahren sind die Krankenkassen verpflichtet, gemäß $§ 69$ Abs. 4 SGB IV in geeigneten Bereichen eine Kosten- und Leistungsrechung (KLR) einzuführen, insbesondere um die Struktur der Verwaltungskosten transparenter zu gestalten (Klemz 2004, S. 103). Für Krankenkassen stellt die KLR eine hinreichende, aber nicht zwingend notwendige Voraussetzung für den BSC-Einsatz dar. Hinreichend, da vielfältige Informationen für das Aufstellen von Kennzahlen nötig sind. Nicht zwingend notwendig, da Informationen für Kennzahlen auf anderen Wegen generiert werden können (und auch müssen). Dennoch ist davon auszugehen, dass Krankenkassen mit BSC-Einsatz sich bei der Implementierung der KLR als Vorreiter erweisen.

7 Derzeit gibt es etwa 237 Krankenkassen, die in sieben Kassenarten organisiert sind (GKV 2008): 7 Angestellten-Krankenkassen (AKK), 2 Arbeiter-Ersatzkassen (EKK), 17 Ortskrankenkassen (AOK), 199 Betriebskrankenkassen (BKK), Knappschaft, 16 Innungskrankenkassen (IKK), 9 Landwirtschaftliche Krankenkassen (LKK). Zum Zeitpunkt der empirischen Erhebung gab es eine weitere Kassenart, die See-Krankenkasse. Diese schloss sich zum 1. Januar 2008 mit der Knappschaft zusammen. 
Weiterhin stellt sich die Frage, ob Krankenkassen ohne BSC-Einsatz auch Kennzahlensysteme anwenden. Umfassende Kennzahlensysteme scheinen in vielen Krankenkassen bislang zu fehlen (Schlösser/Schreyögg 2005, S. 324). Demnach ist auch hier zu vermuten, dass Kassen ohne BSC-Einsatz in geringerem Umfang betriebswirtschaftliche Instrumente einsetzen, d. h. bei der Steuerung ihrer Organisation weniger auf Kennzahlensysteme bauen.

Letztlich ist davon auszugehen, dass Krankenkassen mit BSC-Einsatz umfassende Anreizsysteme für Führungskräfte aber auch Mitarbeiter implementiert haben, um durch individuelle Zielvereinbarungen die Strategieumsetzung der BSC sicherzustellen (Schwertner et al. 2005, S. 38). Im Vertrieb scheinen die Krankenkassen breit auf Anreizsysteme zu setzen (Marburger/Marburger 1997, S. 68). Ungewiss ist hingegen, ob Kassen ohne BSC-Einsatz auch eine hohe Implementierung von Anreizsystemen bei Mitarbeitern und Führungskräften besitzen. Daher wird an dieser Stelle hypothetisiert, dass Krankenkassen mit BSC-Einsatz umfassender mit Anreizsystemen arbeiten bzw. die BSC deren Implementierung fördert.

Die vierte Forschungsfragestellung widmet sich schließlich dem Aufbau und der Ausgestaltung der BSC in Krankenkassen. Dabei werden die Perspektiven, Implementierungsebenen, Kennzahlen und Ursache-Wirkungsbeziehungen analysiert. Wie oben bereits aufgezeigt liegen in der Literatur nur sehr wenige Erfahrungen zur BSC in Krankenkassen aus der Praxis vor. Theoretische Arbeiten zeigen erste Anhaltspunkte einer spezifischen Anpassung des BSC-Konzeptes an die Krankenkassen (Schlösser/Schreyögg 2005; Moos/Brüggemann 2006). Aufgrund des Non-Profit-Charakters der gesetzlichen Krankenkassen und der besonderen Rahmenbedingungen im GKV-Markt ist daher mit spezifischen Anpassungen bei den Kennzahlen, aber auch in den Perspektiven und deren hierarchischer Anordnung zu rechnen. Letzteres wurde beispielsweise in einer Erhebung zur BSC in deutschen Krankenhäusern untersucht. Danach befand sich in fast nur jedem zehnten Fall die Finanzperspektive weiterhin allein an oberster Stelle (Ahn/Reuter 2005, S. 615).

Tab. 1 gibt noch einmal zusammenfassend die Forschungsfragestellungen und die zugehörigen zentralen Forschungshypothesen wieder. 
F1) Wie ist der Stand der Anwendung und der Bekanntheitsgrad der BSC in gesetzlichen Krankenkassen? - Der Verbreitungsgrad der BSC in Krankenkassen bewegt sich im Bereich von 20\%-30\%.

F2) Was charakterisiert Krankenkassen mit BSC-Einsatz und hat die BSC-Einführung einen Einfluss auf die Entwicklung der Versichertenzahlen?

- Größere Krankenkassen setzen die BSC häufiger ein als kleinere Kassen.

- Der relative Anteil an Krankenkassen mit BSC-Einsatz unterscheidet sich nicht nach Kassenarten.

- Krankenkassen mit BSC-Einsatz weisen eine im Durchschnitt günstigere Entwicklung der Versichertenzahlen auf als diejenigen ohne.

F3) Arbeiten Krankenkassen ohne BSC-Einsatz weniger mit (BSC-nahen) Steuerungsinstrumenten als diejenigen mit BSC-Einsatz?

- Strategien werden in Krankenkassen mit BSC-Einsatz deutlich häufiger formuliert als in denen ohne.

- Krankenkassen mit BSC-Einsatz haben die KLR umfangreicher implementiert als diejenigen ohne.

- Krankenkassen mit BSC-Einsatz besitzen in höherem Umfang Kennzahlensysteme zur Steuerung der Organisation als diejenigen ohne.

- Krankenkassen mit BSC-Einsatz arbeiten umfassender mit Anreizsystemen als diejenigen ohne.

F4) Wie gestalten sich der Aufbau und die Ausgestaltung der BSC in Krankenkassen?

- Der Aufbau und die Ausgestaltung der BSC in gesetzlichen Krankenkassen weist spezifische Anpassungen zum BSC-Grundkonzept auf, insbesondere was die Kennzahlen betrifft, aber auch in der Perspektivenwahl und deren hierarchischer Anordnung.

Tab. 1: Forschungsfragestellungen und -hypothesen

Quelle: $\quad$ Eigene Darstellung

\section{Empirische Untersuchung}

\section{Vorgehensweise}

Zur Bearbeitung der ersten drei der oben genannten vier Forschungsfragen wurde ein zweiseitiger Fragebogen eingesetzt. Der Fragebogen enthielt allgemeine Fragen zu Planungs- und Steuerungsinstrumenten wie z. B. „Hat Ihre Krankenkasse Vergütungssysteme/Anreizsysteme für Führungskräfte/Mitarbeiter, die mit individuellen Zielvereinbarungen verknüpft sind?“ oder „Arbeitet Ihre Krankenkasse im Rahmen der strategischen bzw. operativen Planung und Steuerung mit einer Balanced Scorecard, oder sind Sie in der Phase der Einführung?“. Die vierte Fragestellung zum Aufbau und der Ausgestaltung der BSC wurde im Rahmen von Experteninterviews mit Hilfe eines strukturierten Gesprächsleitfadens untersucht.

Vor dem Einsatz der Erhebungsinstrumente wurden Fragebogen und Leitfaden einem Pretest mit Experten aus verschiedenen gesetzlichen Krankenkassen unterzogen, der zu geringfügigen Modifikationen der Instrumente geführt hat. Die Experteninterviews wurden auf Band aufgezeichnet, in einer Zusammenfassung niedergeschrieben und den Gesprächspartnern noch einmal zur Validierung vorgelegt. Die verwendeten Fragen der Erhebungsinstrumente orientierten sich an empirischen Untersuchungen zur BSC in der Industrie und im Krankenhaussektor. ${ }^{8}$

8 Speckbacher/Bischof 2000; Ruhtz 2001; Horváth 2001; Zdrowomyslaw et al. 2003; Horváth \& Partners 2005; Ahn/Reuter 2005. 
Der Erhebungsablauf der empirischen Untersuchung gliederte sich in zwei Phasen: Im Rahmen einer schriftlichen Vollerhebung wurde im Juli 2006 der Fragebogen mit dem begleitenden Anschreiben an die Vorstände von 247 gesetzlichen Krankenkassen postalisch gesandt. ${ }^{9}$ Die Vorstände oder gegebenenfalls Mitarbeiter mit entsprechenden Kenntnissen wurden gebeten, den beigelegten Fragebogen auszufüllen und im angefügten Rückumschlag zurückzusenden. Weitere Erinnerungsschreiben folgten im August und September. Letzterem wurde zusätzlich ein Kurz-Fragebogen beigelegt. Zweck dieses Fragebogens war es, eine möglichst hohe Rücklaufquote bei der Frage nach dem BSCEinsatz zu erhalten. Die schriftliche Untersuchung diente auch zur Identifikation von potenziellen Ansprechpartnern für eine inhaltliche Analyse der BSC. Bei der zweiten Erhebungsphase wurden zur Analyse der BSC in gesetzlichen Krankenkassen von Dezember 2006 bis Februar 200713 leitfadengestützte Experteninterviews geführt. Davon erfolgten elf telefonisch, die restlichen persönlich.

Die Erhebungsmethode der Expertengespräche wurde aufgrund der geringen Anzahl von Krankenkassen mit BSC-Anwendung, der Heterogenität der Kassenlandschaft und des Pilot-Charakters der Studie gewählt. Der Vorzug dieser Erhebungsmethode gegenüber einem Fragebogen lag darin, dass durch die offene Gesprächsführung und die Erweiterung von Antwortspielräumen der Bezugsrahmen des Befragten bei der Fragenbeantwortung miterfasst werden konnte, um so einen Einblick in die Erfahrungshintergründe und die Schwerpunktsetzung des Befragten zu erhalten (Schnell et al. 2005, S. 319 ff.). Die Auswertungsstrategie bei den Experteninterviews orientierte sich an einer bewährten Konzeption von Meuser und Nagel (Meuser/Nagel 1991, S. 455 ff.).

Die in der schriftlichen Befragung gewonnenen Daten wurden in zweifacher Form von verschiedenen Personen in die MS-Access Datenbank eingegeben und abgeglichen. Die Auswertung des Fragebogens erfolgte mit dem SAS Statistik-Software-Paket. Neben Häufigkeitsauszählungen wurden statistische Analyseverfahren wie der Chi-Quadrat-Test und die logistische Regression eingesetzt.

\section{Ergebnisse der schriftlichen Befragung}

\section{a) Rücklauf und Repräsentativität}

Bis zum Abschluss der schriftlichen Befragung im Oktober 2006 antworteten 84 der 247 angeschriebenen gesetzlichen Krankenkassen, was einer Rücklaufquote von $34 \%$ entspricht (siehe Abb. 1). ${ }^{10}$

9 Die Anzahl und Anschriften der gesetzlichen Krankenkassen wurden im Mai 2006 über das Internet auf den Seiten der GKV und einzelner Kassenverbände recherchiert.

10 Vier der 84 Krankenkassen haben im Rahmen des letzten Anschreibens einen beigelegten Kurz-Fragebogen mit nur drei Fragen (BSC Anwendung, Versichertenanzahl und Kassenart) ausgefüllt. Daher lagen für einige der Studienfragen nur max. 80 Fragebögen vor. 


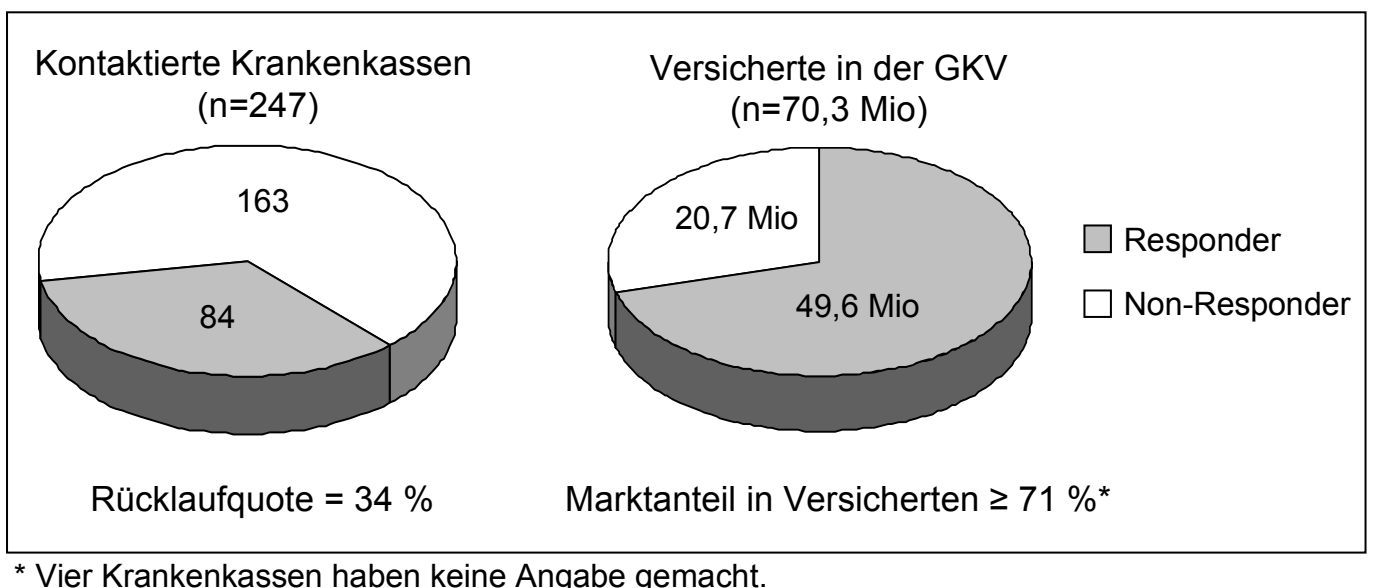

Abb. 1: Fragebogenrücklauf und Marktanteil der teilnehmenden Krankenkassen Quelle: $\quad$ Eigene Darstellung

Aus der Versichertenzahl der teilnehmenden Krankenkassen in Relation zur Versichertenzahl der GKV lässt sich der Marktanteil der Studie beziffern. Zusammen genommen versichern die 80 Studienteilnehmer (vier haben dazu keine Angabe gemacht) 49,6 Millionen Menschen und damit $71 \%$ des gesamten GKV-Marktes. Die Streuung der Versichertenzahl reicht dabei von 1.750 bis sieben Millionen. Die durchschnittliche Kassengröße der Studie liegt bei rund 620.000 Versicherten, die aller gesetzlichen Krankenkassen bei nur rund 285.000. Bei den teilnehmenden Krankenkassen waren alle Kassenarten vertreten. Mit $24 \%$ beteiligten sich die Betriebskrankenkassen (BKK) prozentual am geringsten und mit $86 \%$ die Angestelltenkrankenkassen (AKK) am häufigsten. Bei 11 Krankenkassen fehlen die Angaben zur Kassenart. Davon ist aufgrund der abgegebenen Versichertenzahl vermutlich eine den AOKn (Allgemeine Ortskrankenkassen), die weiteren den BKKn zuzuordnen.

\section{b) Bekanntheitsgrad und Stand der Anwendung}

Der Bekanntheitsgrad des BSC-Konzeptes in Krankenkassen liegt bei $85 \%$ (siehe Abb. 2). Nur 13 (15\%) Krankenkassen kennen das BSC-Konzept nicht. 22 der 84 teilnehmenden Krankenkassen arbeiten mit der BSC oder sind in der Phase der Einführung. Eine hat die BSC-Anwendung bereits abgebrochen. Mehr als ein Viertel (26\%) aller gesetzlichen Krankenkassen des Studienkollektivs beschäftigten sich demnach aktiv mit der BSC. Der Höhepunkt der BSC-Einführung in den Krankenkassen war in den Jahren 2002-2004, als 75 \% BSCs einführten. Die erste BSC wurde 1998 eingeführt. Im Durchschnitt arbeiten die Krankenkassen seit vier Jahren mit der BSC. Die in der Untersuchung ermittelten Zahlen belegen damit die Hypothese bezüglich des Verbreitungsgrads der BSC in gesetzlichen Krankenkassen. 


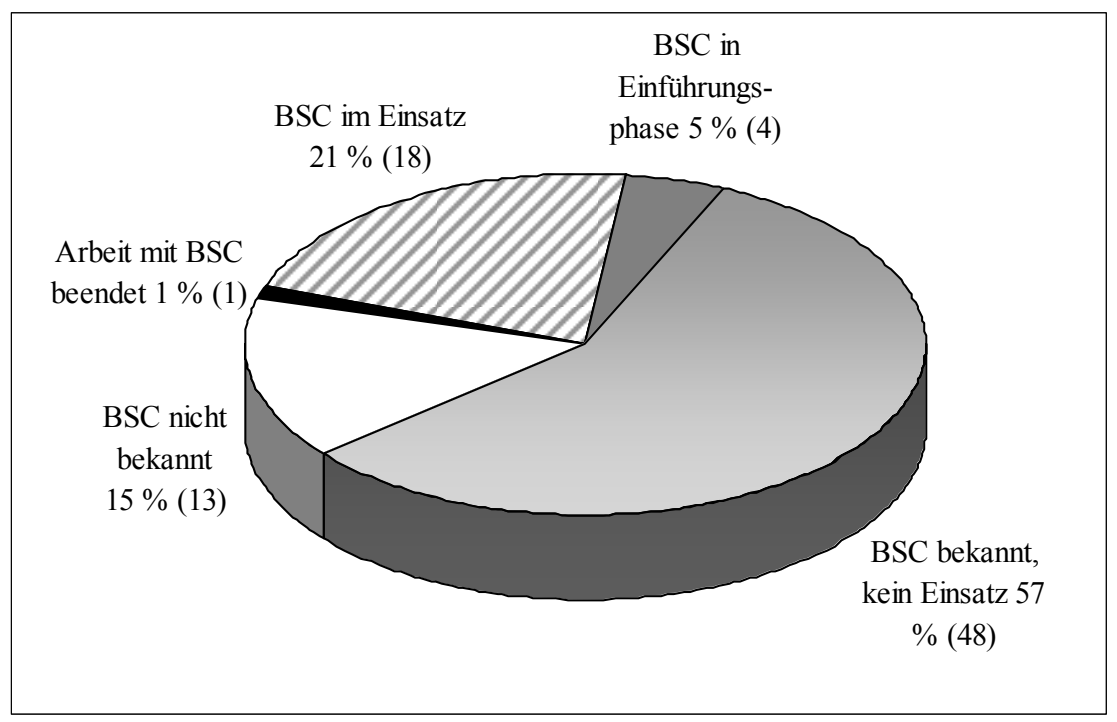

Abb. 2: Bekanntheit und Einsatz der Balanced Scorecard Quelle: $\quad$ Eigene Darstellung

\section{c) Charakteristika der (BSC-)Krankenkassen und Entwicklung der Versichertenzahlen}

Zur Überprüfung der oben aufgestellten Hypothesen, dass größere Krankenkassen die BSC häufiger einsetzen und dass die Kassenart keine Determinante des BSC-Einsatzes ist, wurde eine binäre logistische Regression durchgeführt. ${ }^{11}$ Dabei diente die Variable BSC-Einsatz als abhängige Variable. Die Kassengröße (Anzahl der Versicherten) ging als stetige logarithmierte unabhängige Variable in das Regressionsmodell ein. ${ }^{12}$ Die zweite unabhängige Variable war die Kassenart (ordinale Variable), wobei die BKKn als Referenzwert dienten. ${ }^{13}$

Die Güte des logistischen Regressionsmodells wurde verschiedentlich geprüft. Der Hosmer-Lemeshow-Test kam für das gewählte Regressionsmodell zu keinem signifikanten Ergebnis, was für eine unbedenkliche Modellgültigkeit spricht. Auch bezüglich der Modellrelevanz sind keine Probleme erkennbar. Der Likelihood-Ratio-Test führt in Bezug auf das Gesamtmodell zu einem signifikanten Ergebnis auf $1 \%$-Niveau, so dass das Modell insgesamt eine gute Trennkraft für die Unterscheidung des Einsatzes der BSC aufweist. Mit 79,6\% richtiger eingeordneter Krankenkassen (BSC im Einsatz oder nicht) liefert das Modell eine gute Vorhersagewahrscheinlichkeit. Das Nagelkerke- $\mathrm{R}^{2}$ kann mit 0,31 als akzeptabel angesehen werden (Backhaus et al. 2006, S. 448).

11 Es lag keine Multikollinearität vor. Die Residuenplots wiesen auf einen unabhängigen Fehlerterm hin. Die Voraussetzungen für eine logistische Regression waren demnach gegeben (Backhaus et al. 2006, S. 417 f.).

12 Aufgrund der äußerst heterogenen und rechtsschiefen Verteilung der Kassengröße wurden die Daten logarithmiert, um Ausreißer in die Funktion mit einzubeziehen. Die kategoriale Darstellung der Kassengröße in Tab. 2 wurde zur besseren Anschaulichkeit gewählt.

13 Die BKKn wurden aufgrund der meisten Beobachtungspunkte und damit den kleinsten Konfidenzintervallen als Referenzkategorie gewählt. 
Die Ergebnisse bezüglich der Hypothesen liefert Tab. 2. Deskriptiv zeigt sich mit steigender Kassengröße eine Tendenz zum verstärkten BSC-Einsatz. Der höchste Anteil befindet sich in der Größenkategorie von 250.000 bis unter einer Million Versicherten. Diese Kategorie umfasst insgesamt 13 teilnehmende Krankenkassen (mit und ohne BSCAnwendung). Davon haben 11 (85\%) angegeben, mit der BSC zu arbeiten. Die durchschnittliche Größe der BSC-Krankenkassen liegt mit 931.000 Versicherten deutlich über der mit 502.000 der Nicht-BSC-Krankenkassen (nicht abgebildet). Der Zusammenhang zwischen Kassengröße und BSC-Einsatz erwies sich auf einem 1 \%-Nivau signifikant (Odds Ratio 1,80).

\begin{tabular}{|c|c|c|c|c|}
\hline & & Anzahl & $\begin{array}{l}\text { Regressions- } \\
\text { koeffizient (SF) }\end{array}$ & $\begin{array}{l}\text { Odds Ratio } \\
\text { (KI) }\end{array}$ \\
\hline \multirow[t]{4}{*}{ Kassengröße } & 20-99 Tsd & $4(25 \%)$ & \multirow{4}{*}{$0,58(0,19)^{* *}$} & \multirow{4}{*}{$1,80(1,23-2,62)$} \\
\hline & $100-249 \mathrm{Tsd}$ & $4(29 \%)$ & & \\
\hline & 250-999 Tsd & $11(85 \%)$ & & \\
\hline & 1-7 Mio & $3(23 \%)$ & & \\
\hline \multirow[t]{6}{*}{ Kassenart } & AOK & $3(38 \%)$ & $-1,22(0,99)$ & $0,30(0,04-2,07)$ \\
\hline & AKK/EKK & $3(43 \%)$ & $-2,10(1,51)$ & $0,12(0,01-2,36)$ \\
\hline & BKK & $10(21 \%)$ & - & - \\
\hline & IKK & $4(80 \%)$ & $1,75(1,22)$ & $5,76(0,53-62,39)$ \\
\hline & LKK/Knapp/See & $1(20 \%)$ & $-0,50(0,90)$ & $0,63(0,11-3,62)$ \\
\hline & Anonym & $1(9 \%)$ & $-1,41(1,26)$ & $0,24(0,02-2,87)$ \\
\hline
\end{tabular}

Tab. 2: $\quad$ Charakteristika der BSC-Krankenkassen

Quelle: $\quad$ Eigene Darstellung

Die Hypothese bezüglich der Kassenart, der relative Anteil an Krankenkassen mit BSCEinsatz unterscheidet sich nicht nach Kassenarten, konnte ebenfalls bestätigt werden. Die deskriptiven Unterschiede erwiesen sich nicht als signifikant. In absoluten Zahlen setzen die BKK am häufigsten die BSC ein. Verhältnismäßig liegen die Innungskrankenkassen (IKKn) mit $80 \%$ BSC-Anwendung jedoch deutlich an der Spitze.

Die Entwicklung der Versichertenzahlen innerhalb der vergangenen 12 Monate ist in Tab. 3 für BSC und Nicht-BSC-Krankenkassen (N-BSC) abgebildet. ${ }^{14}$ Auffällig ist hierbei, dass bei $43 \%$ der BSC-Kassen die Versichertenzahlen gesunken sind. Dem entgegen verzeichnen nur $24 \%$ der Nicht-BSC-Kassen einen Mitgliederschwund. Allerdings gaben prozentual etwas mehr BSC-Krankenkassen eine Steigerung der Versichertenzahlen an. Ein signifikanter Unterschied zwischen der Veränderung der Versichertenzahl und dem BSC-Einsatz konnte letztlich nicht festgestellt werden, und damit die diesbezügliche Hypothese, Krankenkassen mit BSC-Einsatz weisen eine im Durchschnitt günstigere

14 Bezüglich der Veränderung der Versichertenzahlen wurden die Krankenkassen nur danach befragt, ob diese „gesunken“, „gleichgeblieben“ oder „gestiegen“ sind. Die tatsächliche Veränderung der Versichertenzahl ist nicht bekannt. 
Entwicklung der Versichertenzahlen auf als diejenigen ohne, nicht verifiziert werden konnte.

\begin{tabular}{|l|c|c|}
\hline $\begin{array}{l}\text { Entwicklung der Versicherten- } \\
\text { zahl (12 Monate) }\end{array}$ & BSC & N-BSC \\
\hline gesunken & $9(43 \%)$ & $14(24 \%)$ \\
\hline gleichgeblieben & $2(10 \%)$ & $20(34 \%)$ \\
\hline gestiegen & $10(48 \%)$ & $25(42 \%)$ \\
\hline Signifikanz $\left(\mathrm{Chi}^{2}\right)$ & \multicolumn{2}{|c|}{ n.s. } \\
\hline
\end{tabular}

* Insgesamt vier fehlende Werte.

Tab. 3: Entwicklung der Versichertenzahlen

Quelle: $\quad$ Eigene Darstellung

\section{d) Anwendung BSC-naher Steuerungsinstrumente}

Da die BSC ein Instrument zur Umsetzung der Unternehmensstrategie ist, besitzen konsequenterweise alle Krankenkassen mit BSC-Anwendung eine formulierte Strategie (siehe Tab. 4). Nicht-BSC-Krankenkassen hingegen folgen nur zu $66 \%$ einer formulierten Strategie, was sich als hoch signifikanter Unterschied $\left(\mathrm{Chi}^{2}\right.$-Test: $\left.\mathrm{p}<0,01\right)$ erweist und damit die diesbezügliche Hypothese bestätigt. Was den Inhalt der Strategien betrifft, gibt es kaum Abweichungen zwischen BSC- und Nicht-BSC-Krankenkassen. An oberster Stelle der Strategieformulierung finden sich (regionales und qualitatives) Wachstum/ Versichertenentwicklung sowie Kundenorientierung/Service, gefolgt von Finanzen/Wirtschaftslage und Qualitä/Leistungen (offene Fragestellung). Die meisten Krankenkassen wollen dabei insbesondere das regionale Umfeld oder Segment (z. B. Handwerk) bedienen und streben ein „qualitatives“ Wachstum, also ein Wachstum mit möglichst gesunden und einkommensstarken Versicherten (so genannte ,gute Risiken“), an.

Die Kosten- und Leistungsrechnung (KLR) haben $90 \%$ der BSC-Krankenkassen implementiert. Umfassend eingeführt, d. h. über den Verwaltungsbereich hinaus, ist die KLR allerdings nur bei $14 \%$ der BSC-Krankenkassen (nicht in der Tabelle dargestellt). Rund $30 \%$ weniger kommt die KLR bei Nicht-BSC-Krankenkassen zur Anwendung, was wiederum einen signifikanten Unterschied zu den BSC-Krankenkassen darstellt. Damit bestätigt sich die Hypothese, dass Krankenkassen mit BSC-Einsatz die KLR umfangreicher implementiert haben als diejenigen ohne.

Auch beim Einsatz von Kennzahlensystemen zeigt sich (konsequenterweise) ein signifikanter Unterschied zwischen Krankenkassen mit und ohne BSC-Anwendung, was erneut die aufgestellte Hypothese bestätigt. Immerhin $54 \%$ der Krankenkassen ohne BSCEinsatz arbeiten mit finanziellen und nicht-finanziellen Kennzahlen. Bei $21 \%$ der Krankenkassen werden keinerlei Kennzahlen gebildet. Eine BSC-Krankenkasse (5\%) gab an, nur finanzielle Kennzahlen anzuwenden. Dies widerspricht der BSC-Idee - der Ausge- 
wogenheit zwischen finanziellen und nicht-finanziellen Parametern - und ist damit nicht nachvollziehbar. ${ }^{15}$

\begin{tabular}{|c|c|c|c|c|}
\hline Strategie & \multicolumn{2}{|r|}{ BSC } & \multicolumn{2}{|c|}{ N-BSC } \\
\hline vorhanden & \multicolumn{2}{|r|}{$100 \%$} & \multicolumn{2}{|c|}{$66 \%$} \\
\hline Signifikanz $\left(\mathrm{Chi}^{2}\right)$ & \multicolumn{4}{|c|}{$p<0,01$} \\
\hline KLR & \multicolumn{2}{|r|}{ BSC } & \multicolumn{2}{|c|}{ N-BSC } \\
\hline vorhanden & \multicolumn{2}{|r|}{$90 \%$} & \multicolumn{2}{|c|}{$63 \%$} \\
\hline Signifikanz $\left(\mathrm{Chi}^{2}\right)$ & \multicolumn{4}{|c|}{$p<0,05$} \\
\hline Kennzahlensystem & \multicolumn{2}{|c|}{ Finanziell/nicht-Fin. } & Finanziell & Kein \\
\hline BSC & \multicolumn{2}{|c|}{$95 \%$} & $5 \%$ & - \\
\hline N-BSC & \multicolumn{2}{|c|}{$54 \%$} & $25 \%$ & $21 \%$ \\
\hline Signifikanz $\left(\mathrm{Chi}^{2}\right)$ & \multicolumn{4}{|c|}{$p<0,01$} \\
\hline \multirow[t]{2}{*}{ Anreizsystem } & \multicolumn{2}{|c|}{ Mitarbeiter } & \multicolumn{2}{|c|}{ Führungskräfte } \\
\hline & kaum & überwiegend & kaum & überwiegend \\
\hline BSC & $71 \%$ & $29 \%$ & $43 \%$ & $57 \%$ \\
\hline N-BSC & $81 \%$ & $19 \%$ & $63 \%$ & $37 \%$ \\
\hline Signifikanz (Chi²) & \multicolumn{2}{|r|}{ n.s. } & \multicolumn{2}{|c|}{ n.s. } \\
\hline
\end{tabular}

Tab. 4: Betriebswirtschaftliche Steuerungsinstrumente in Krankenkassen Quelle: $\quad$ Eigene Darstellung

Anreizsysteme mit individuellen Zielvereinbarungen, die Vergütungsbestandteile einschließen, sind im Bereich der Mitarbeiter von Krankenkassen sowohl bei BSC- als auch bei Nicht-BSC-Kassen kaum verbreitet. Bei Führungskräften haben immerhin $57 \%$ der BSC-Krankenkassen ein an Zielvereinbarungen gekoppeltes Anreizsystem. Insgesamt sind die Unterschiede in der Ausgestaltung der Anreizsysteme bei den Krankenkassen eher gering und nicht signifikant. Insofern kann die Hypothese, dass Krankenkassen mit BSC-Einsatz umfassender mit Anreizsystemen arbeiten als diejenigen ohne, nicht verifiziert werden.

\section{Ergebnisse der Expertenbefragung}

\section{a) Beschreibung der Experteninterviews}

Der Aufbau und die Ausgestaltung der BSC (vierte Forschungsfragestellung) bei den gesetzlichen Krankenkassen wurde anhand der geführten Experteninterviews untersucht. Im

15 Die Krankenkasse konnte nicht für ein Expertengespräch gewonnen werden und eine Überprüfung der Angabe war nicht möglich. 
Rahmen der schriftlichen Untersuchung konnten hierfür potenzielle Interviewpartner ermittelt werden. Von den 22 gesetzlichen Krankenkassen mit BSC-Anwendung antworteten zwei anonym. Daher wurden 20 Krankenkassen, die mit der BSC arbeiten, zur Durchführung von Interviews kontaktiert. Davon konnten 13 Experten für Gespräche gewonnen werden. Die restlichen Krankenkassen haben die Teilnahme verweigert oder sich trotz mehrmaliger Anfragen nicht zurückgemeldet. Bei einer der teilnehmenden Krankenkassen wurde die BSC nicht fertiggestellt, eine weitere befindet sich in der Phase der Einführung. Daher werden diese Interviews nur bei einzelnen Fragestellungen, wo eine sinnvolle Interpretation möglich ist, herangezogen.

Die Aufgabengebiete der teilnehmenden Experten liegen vorwiegend im Bereich des Controllings und der Unternehmensentwicklung, aber auch des Vorstands oder des Risikomanagements. Die Verteilung der Interviews streute breit über die verschiedenen Kassenarten. Die kleinste teilnehmende Krankenkasse versichert rund 55.000 Personen, die größte über sechs Millionen. Die durchschnittliche Kassengröße der an der Expertenbefragung teilnehmenden Krankenkassen liegt bei rund 954.000. Der Durchschnitt aller 22 BSC-Krankenkassen liegt mit 931.000 Versicherten nur leicht darunter. Die Interviewdauer betrug zwischen 40 und 70 Minuten.

\section{b) Aufbau und Ausgestaltung der Balanced Scorecard}

Im Folgenden werden die Ergebnisse der Experteninterviews zur BSC in gesetzlichen Krankenkassen anhand der Perspektiven, Implementierungsebenen, Kennzahlen und Ursache-Wirkungsbeziehungen vorgestellt.

\section{aa) Perspektiven}

Die Auswahl der Perspektiven der BSC in gesetzlichen Krankenkassen ist in Tab. 5 gegeben. Auf der linken Seite der Tabelle wird der „klassische“ Aufbau der BSC, auf der rechten Seite die Alternativen abgebildet. Acht der 13 Krankenkassen (62\%) arbeiten mit den vier klassischen Perspektiven Finanzen, Kunden, Prozesse und Mitarbeiter. Drei weitere Krankenkassen verwenden ebenfalls vier Perspektiven, haben jedoch eine neue Perspektive kreiert (und die Prozessperspektive eliminiert) bzw. ihre Anordnung verändert. ${ }^{16}$ Nur zwei BSCs der Krankenkassen (15\%) beinhalten fünf Perspektiven. Die Perspektive der Kunden wird von den Krankenkassen in unterschiedlicher Tragweite behandelt. Neben den Versicherten beziehen einzelne Krankenkassen auch die Arbeitgeber der Versicherten und die Leistungserbringer explizit in diese Perspektive mit ein.

16 Anmerkung: Die Prozessperspektive wurde zwei BSCs zwar entnommen, findet sich jedoch größtenteils in der Perspektive Mitarbeiter bzw. interne Ressourcen inhaltlich wieder. 


\begin{tabular}{|c|c|c|c|c|c|c|}
\hline & Klassisch $(8 x)$ & \multicolumn{5}{|c|}{ Alternativen (5) } \\
\hline 1 & Finanzen & Politik & Finanzen & Kunden & Finanzen & Kunden/Markt \\
\hline 2 & Markt/Kunden & Kunden & Kunden/Markt & Ökonomie & Kunden & Mitarbeiter \\
\hline 3 & Prozesse & Finanzen & $\begin{array}{l}\text { Medizinische Versorgung } \\
\text { \& Leistungsangebot }\end{array}$ & Prozesse & Prozesse & Finanzen \\
\hline 4 & $\begin{array}{l}\text { Mitarbeiter/Poten- } \\
\text { zial/Entwicklung }\end{array}$ & Mitarbeiter & Interne Ressourcen & $\begin{array}{l}\text { Innovation } \\
\text { \& Lernen }\end{array}$ & Mitarbeiter & Prozesse \\
\hline 5 & & & & & $\begin{array}{l}\text { Identität \& } \\
\text { Vision }\end{array}$ & Produkte \\
\hline
\end{tabular}

Tab. 5: Aufbau (Perspektiven) der BSC gesetzlicher Krankenkassen

Quelle: $\quad$ Eigene Darstellung

Perspektiven, die zusätzlich in die BSC aufgenommen wurden bzw. eine Perspektive ersetzt haben, sind die Perspektiven „Politik“, „Identität \& Vision“, „Medizinische Versorgung \& Leistungsangebot“" sowie „Produkte“. Die beiden zuletzt genannten Perspektiven decken sich teilweise inhaltlich, indem sie jeweils die Möglichkeiten der selektiven Vertragsgestaltung bzw. die Weiterentwicklung von Versorgungsangeboten einbeziehen. Wie in den Gesprächen mit den Experten zu erfahren war, denken einige Krankenkassen insbesondere über das Hinzuziehen weiterer Perspektiven wie „Produkte/Leistungen“ oder auch „Politik“ nach.

Zwei Krankenkassen stellen die Kundenperspektive an die Spitze der BSC. Die Finanzperspektive befindet sich zum Teil auch an zweiter bzw. dritter Stelle der BSC, während die Prozessperspektive einmal an vierter Stelle der BSC genannt wird. Zu berücksichtigen ist dabei allerdings, dass einige der Krankenkassen auf eine streng hierarchische Anordnung der Perspektiven verzichten bzw. die Perspektiven als gleichwertig einschätzen. Darin zeigt sich bereits, wie später noch zu sehen sein wird, dass einige der Krankenkassen auf eine umfassende Verbindung der Beziehungen mit Ursache-Wirkungsketten verzichtet haben.

\section{bb) Implementierungsebenen}

Die gesetzlichen Krankenkassen weisen aufgrund der historischen Prägung und unterschiedlichen Kassengröße Differenzen im Organisationsaufbau auf. Während große Krankenkassen teilweise über mehrere hundert Geschäftsstellen verfügen, haben kleine Krankenkassen oft nur einige wenige, im Extremfall einer Direkt-Krankenkasse sogar keine Geschäftsstellen. Auch bezüglich der Geschäfts- bzw. Unternehmensbereiche (z. B. Leistung, Verträge, Vertrieb, Finanzen, Marketing) oder der Stabstellen (z. B. Unternehmensentwicklung, Controlling) liegt ein hoher Differenzierungsgrad vor. Dennoch hat sich im Rahmen der Experteninterviews eine gewisse Struktur im Aufbau der Krankenkassen manifestiert. Nach dem Vorstand beinhaltet die zweite Unternehmensebene überwiegend die Geschäftsbereiche, Regionaldirektionen und im Grunde auch die Stabstellen (obwohl diese für gewöhnlich allen Bereichen zuarbeiten). Als dritte Ebene folgen die 
jeweiligen Abteilungen der Geschäftsbereiche, auf der vierten Ebene die Teams und dann die Mitarbeiter.

Den Implementierungsgrad der BSC in gesetzlichen Krankenkassen zeigt Tab. 6. Auf der Unternehmensebene besitzen alle Krankenkassen eine BSC. Die Perspektiven, die die Krankenkassen auf den weiteren Ebenen benutzen, sind mit wenigen Ausnahmen dieselben, wie die der jeweiligen Unternehmens-BSC. Beispielsweise wurde in einer Krankenkasse für die BSC im ambulanten Bereich auf die Perspektive „Kunde/Markt“ verzichtet, da die Prozesse meist nicht beim Kunden enden. Im Gegenzug wurde die Perspektive „interne Ressourcen“ weiter aufgebrochen. Andere Krankenkassen haben verschiedene Schwerpunkte in ihren Regionaldirektionen. Prüfte man dort beispielsweise die Krankenhausrechnungen, so findet dies seinen Niederschlag in der BSC (Kennzahl z. B.: Steigerung der eingesparten Beträge).

\begin{tabular}{|l|c|c|c|c|c|c|c|c|c|c|c|}
\hline \begin{tabular}{|l|c|} 
Krankenkasse \\
BSC-Ebene
\end{tabular} & a & b & c & d & e & f & g & h & i & j & k \\
\hline Unternehmen & 1 & 1 & 1 & 1 & 1 & 1 & 1 & 1 & 1 & 1 & 1 \\
\hline Geschäftsbereiche $^{\#}$ & - & 1 & 8 & 4 & 30 & 12 & - & 9 & 10 & 7 & 10 \\
\hline Abteilungen & - & 7 & 17 & 28 & - & 7 & - & - & - & - & 16 \\
\hline Teams & - & - & $100^{*}$ & 84 & - & - & - & - & - & - & - \\
\hline Mitarbeiter & - & - & $750^{*}$ & - & - & - & - & - & - & - & - \\
\hline Summe BSCs & $\mathbf{1}$ & $\mathbf{9}$ & $\mathbf{8 7 6}$ & $\mathbf{1 1 7}$ & $\mathbf{3 1}$ & $\mathbf{2 0}$ & $\mathbf{1}$ & $\mathbf{1 0}$ & $\mathbf{1 1}$ & $\mathbf{8}$ & $\mathbf{2 7}$ \\
\hline
\end{tabular}

* Schätzungen

\# Inklusive Geschäftsstellen/Regionaldirektionen und Stabstellen

Tab. 6: Implementierungsebenen und BSC-Systeme gesetzlicher Krankenkassen Quelle: $\quad$ Eigene Darstellung

Auf der zweiten Ebene der Geschäftsbereiche, Regionaldirektionen und Stabstellen besitzen rund $82 \%$ der Krankenkassen noch einzelne BSCs, wobei sich deren Anzahl zwischen einer und 30 bewegt. Bei knapp der Hälfte der Krankenkassen wird die BSC neben der Steuerung des Gesamtunternehmens und der Geschäftsbereiche auch zur Steuerung der Abteilungen und der Teams - zu immerhin noch $18 \%$ - eingesetzt. Insgesamt zeigt sich, dass mit einer tieferen Einsatzebene die Häufigkeit der BSC sinkt.

Die Summe der BSCs erstreckt sich von nur einer BSC bis hin zu 876 BSCs in einer Krankenkasse. In der Praxis arbeiten daher viele Krankenkassen mit einem System von mehreren BSCs. Die Tiefe der Implementierung wie auch die Summe der BSCs sind unabhängig von der Kassenart und Kassengröße. Ferner besteht keine unmittelbare Beziehung zwischen dem Einführungsjahr und der Anzahl der BSCs. Vielmehr stellte sich in den Interviews heraus, dass die Philosophie der BSC unterschiedlich gelebt wird. In einigen Krankenkassen wird die BSC bis auf Mitarbeiterebene kommuniziert, andere behalten dies dem Führungskreis vor. Als wesentliches Argument gegen eine tiefe Implementierung wurde von den Interviewpartnern die negativ eingeschätzte Relation zwischen Aufwand und Ertrag angeführt. 


\section{cc) Kennzahlen}

Die gesetzlichen Krankenkassen beschränken sich in der Regel auf 20-25 Kennzahlen in ihren BSCs. Eine Zusammenfassung der zentralen, in der Untersuchung gewonnenen Ziele und Kennzahlen einer BSC in gesetzlichen Krankenkassen gibt Tab. 7. Dabei werden Kennzahlen aus allen acht von den Krankenkassen eingesetzten Perspektiven aufgezeigt. ${ }^{17}$ Während die oberen vier Perspektiven bei der überwiegenden Anzahl von Kassen eingesetzt werden, kommen die unteren nur teilweise zum Einsatz (siehe auch Tabelle 5). In der Finanzperspektive befinden sich Kennzahlen zum Beitragssatz, der Abweichung der tatsächlichen zu den standardisierten Leistungsausgaben, den Verwaltungskosten, der Rückstandsquote der Beiträge, der Rücklagenanteil oder die Rückführung des Betriebsmitteldefizits.

Die dominanteste Kennzahl im „Kunden/Markt“-Bereich ist die Entwicklung der Versichertenzahlen. Während einige der Krankenkassen sich bemühen, die Abwanderung von Versicherten zu stoppen, versuchen andere Krankenkassen die Versichertenanzahl zu steigern, um Größen- und Verbundeffekte zu erzielen. „Qualitatives“ Wachstum bedeutet dabei, insbesondere so genannte „gute Risiken“ bzw. Versicherte mit einem hohen Deckungsbeitrag zu versichern. Weitere Kennzahlen bilden unter anderem die Kundenzufriedenheit, die Schnelligkeit im Service (Erledigung innerhalb 48 h) oder der Bekanntheitsgrad.

Die Kennzahlen in den Perspektiven „Prozesse“ und „Mitarbeiter“ sind weniger kassenspezifisch. Nur bei der Rechnungsprüfung oder der Betreuungsquote für Versicherte zeigt sich der direkte Kassenbezug. Wie unterschiedlich einsatzfähig manche Kennzahlen sind, lässt sich an der Kennzahl „Fluktuationsquote der Mitarbeiter“ beschreiben. Eine Krankenkasse aus einer eher strukturschwachen Region berichtete, dass diese Kennzahl dort keinerlei Relevanz besitze, da es aufgrund hoher Arbeitslosigkeit praktisch keine Fluktuation gebe.

17 Perspektiven mit unterschiedlicher Bezeichnung aber sehr ähnlichen Inhalten wie „Mitarbeiter“ und „Interne Ressourcen" sind als eine Perspektive angeführt. 


\begin{tabular}{|c|c|}
\hline Finanzen/Ökonomie & Kunden/Markt \\
\hline $\begin{array}{l}\text { - Wettbewerbsfähiger Beitragssatz } \\
\text { - Diff. zu standardisierten Leistungsausgaben } \\
\text { - Rückstandsquote je Mitglied/Beiträge } \\
\text { - Rücklagenanteil in Monatsausgaben } \\
\text { - Verwaltungskostenanteil am Gesamtbudget/ } \\
\text { Leistungsausgaben } \\
\text { - Rückführung Betriebsmitteldefizit } \\
\text { - DMP-Versichertenjahre/Teilnehmerquote DMP } \\
\text { - } \varnothing-\text {-Gehaltsaufwendungen je Versicherten } \\
\text { - Zinserträge }\end{array}$ & $\begin{array}{l}\text { - Mitglieder/Versichertenentwicklung (Qualitativ, } \\
\text { - Harktanteil) } \\
\text { - Kaltequote } \\
\text { - Anzundenzufriedenheit (Versicherte/Arbeitgeber) } \\
\text { - Hoher Servicegrad } \\
\text { - Großes Leistungsangebot (Neuproduktrate) } \\
\text { - Anzahl der Versicherten in Programmen } \\
\text { - Positives Image } \\
\text { - Bekanntheitsgrad }\end{array}$ \\
\hline Prozesse/Organisation & Mitarbeiter/Interne Ressourcen \\
\hline $\begin{array}{l}\text { - Optimierung der Arbeits- und Geschäftsprozes- } \\
\text { se (Fehlerpotenzial, Zeit pro Fallbearbeitung) } \\
\text { - Betreuungsquoten (Versicherte, Arbeitgeber } \\
\text { pro Sachbearbeiter) } \\
\text { - Verbesserungswesen (Anzahl der Vorschläge, } \\
\text { Wert der Einsparungen) } \\
\text { - Anzahl durchgeführter Qualitätszirkel } \\
\text { - (Automatisierte) Rechnungsprüfung ausbauen } \\
\text { - Time to Market neuer Produkte } \\
\text { - Optimale IT-Unterstützung am Arbeitsplatz }\end{array}$ & $\begin{array}{l}\text { - Personalentwicklung (Schulungstage je Mitar- } \\
\text { beiter, jährliches Coaching) } \\
\text { - Anzahl geförderter Personen im Unternehmen } \\
\text { - Krankheits-/Fluktuationsquote der Mitarbeiter } \\
\text { - Zufriedenheitsindex Mitarbeiter } \\
\text { - Realisationsquote Ideen } \\
\text { - Weiterentwicklung der IT } \\
\text { - Identifikationsindex der Mitarbeiter mit } \\
\text { Unternehmen }\end{array}$ \\
\hline Produkte & Med. Versorgung \& Leistungsangebot \\
\hline $\begin{array}{l}\text { - Anzahl in DMP eingeschriebener Versicherter } \\
\text { - Krankengeldquote } \\
\text { - Anzahl neuer Produkte (Service \& Leistungen) }\end{array}$ & $\begin{array}{l}\text { - Einsparungen in Millionen } € \text { ( Weiterentwick- } \\
\text { lung der Versorgung, Kostenmanagement) } \\
\text { - Anzahl von Verträgen } \\
\text { - Verträge in bestimmten Indikationen }\end{array}$ \\
\hline Identität \& Vision & Politik \\
\hline $\begin{array}{l}\text { - Marktführerschaft in Geschäftsfeldern erhalten } \\
\text { - Anzahl der Marketingaktivitäten }\end{array}$ & $\begin{array}{l}\text { - Beitragshöhe } \\
\text { • Höhe der zugewiesenen Bundesmittel }\end{array}$ \\
\hline
\end{tabular}

\section{Tab. 7: Zentrale Ziele und Kennzahlen der BSC in gesetzlichen Krankenkassen} Quelle: $\quad$ Eigene Darstellung

Die neu kreierten Perspektiven „Produkte“ und „Medizinische Versorgung \& Leistungsangebot" greifen den spezifischen Produktrahmen der Krankenkassen auf. Kennzahlen in den Perspektiven betreffen etwa die Anzahl eingeschriebener Versicherter in verschiedenen Programmen, die Anzahl neuer Produkte und Verträge oder die Krankengeldquote. Krankenkassen, die eine solche Leistungsperspektive nicht in ihre BSC aufgenommen haben, arbeiten dennoch mit einzelnen äquivalenten Kennzahlen. So findet sich etwa die Anzahl der Versicherten in Programmen sowohl in der Finanz- als auch in der Kundenperspektive wieder.

Eine Krankenkasse, bei welcher die BSC allerdings nicht vollständig umgesetzt wurde, setzte neben den vier klassischen Perspektiven die Perspektive „Identität und Vision“ ein. Beispielhafte Kennzahlen aus dieser BSC-Perspektive sind die Erhaltung der Marktführerschaft im eigenen Segment oder die Anzahl der Marketingaktivitäten.

Der Einbezug einer Politikperspektive wurde nur von einer Krankenkasse vorgenommen. Dies erklärt sich u. a. aus ihrer Krankenkassenart (Landwirtschaftliche Krankenkasse 
(LKK)), die um die Beibehaltung ihrer Sonderstellung in der GKV bemüht ist. Die Beiträge für Landwirte werden nach einem korrigierten Flächenwert berechnet und nicht, wie in der übrigen GKV, als prozentualer Anteil am Einkommen. Für Rentner und sonstige versicherte Personen, die das 65. Lebensjahr vollendet haben, erhalten die LKKn Zuschüsse aus Mitteln des Bundes. Die Kennzahlen der Politikperspektive spiegeln genau diesen Sachverhalt wider.

Schwierigkeiten bereiten den befragten Krankenkassen die Definition geeigneter und auch messbarer, nicht-finanzieller Kennzahlen. So müssen etwa in der Prozessperspektive Prozesse zunächst beschrieben werden, um diese im weiteren Verlauf messen zu können. Ziele, wie „optimale IT-Unterstützung an jedem Arbeitsplatz“, werden zum Teil in allgemeiner Form gemessen (z. B. Dauer zwischen Anfrage und Erledigung). Der angemessene Einbezug nicht-finanzieller Kennzahlen in die BSC ist häufig mit einem hohen Diskussions- und Messaufwand verbunden.

\section{dd) Ursache-Wirkungsbeziehungen}

Die Verbindung der einzelnen Zielkategorien in Ursache-Wirkungsketten stellt eines der zentralen Elemente der BSC dar (Scheibeler 2004, S. 18; Horváth \& Partners 2004, S. 205). Die Abbildung von Ursache-Wirkungsbeziehungen durch Strategy Maps (Strategielandkarten) trat erst im Laufe der Diskussion um die BSC in Erscheinung (Lawrie/ Cobbold 2004, S. 614). Im Mittelpunkt einer Strategy Map steht nicht die vollständige und umfassende Beschreibung des Geschäftsmodells, sondern die Fokussierung auf strategische Ziele mit hoher Wettbewerbsrelevanz und hoher Handlungsnotwendigkeit und die damit verbundene strategische Kernbotschaft (Gaiser/Wunder 2004, S. 458 f.).

Fast alle gesetzlichen Krankenkassen führen Diskussionen bezüglich der UrsacheWirkungsbeziehungen zwischen den Kennzahlen. Allerdings besprechen etwa zwei Drittel der Krankenkassen diese nur vereinzelt. Die Diskussionen waren dabei meist themengeleitet, insbesondere um festzustellen, wo Zielkonflikte und Widersprüche vorlagen oder Verteilungsaspekte geklärt werden mussten. Ein Beispiel: Eine Reduktion von Satzungsleistungen kann zu Kosteneinsparungen führen, womöglich den Beitragssatz senken und damit potenzielle Kunden anlocken. Gleichzeitig könnte die Kundenzufriedenheit zurückgehen und eine Abwanderung von Versicherten erfolgen.

Rund ein Drittel der Krankenkassen beschäftigt sich ausführlich mit den UrsacheWirkungsbeziehungen. Bei der Ermittlung der Ursache-Wirkungszusammenhänge kommt es in der Praxis jedoch zu erheblichen Schwierigkeiten. Das Zusammenwirken der verschiedenen Messgrößen beruht häufig auf subjektiven Einschätzungen, da die $\mathrm{Zu}-$ sammenhänge der Größen untereinander nur schwer quantifizierbar sind oder mit zeitlicher Verzögerung eintreten. Nur wenige Krankenkassen „quantifizieren“ die Zusammenhänge, indem sie etwa die Dicke der Pfeilverbindung zwischen stark, mittel und wenig differenzieren.

Abbildung 3 zeigt eine mögliche Strategielandkarte einer gesetzlichen Krankenkasse mit den vier klassischen Perspektiven, die sich aus den Gesprächen mit den Experten zu- 
sammenfassen lässt. Die Ursache-Wirkungsbeziehungen bauen dabei von der Potenzialperspektive ausgehend nach oben zur Finanzperspektive auf, wo sie in die Ziele zur Einnahmen- und Ausgabenverbesserung münden.

Weniger als ein Drittel der Krankenkassen bilden die Zusammenhänge in solchen Strategielandkarten ab. Der Zweck besteht darin, ein Verständnis für Abhängigkeiten zwischen einzelnen Zielen zu schaffen. Bei Einführungsveranstaltungen zur BSC werden diese häufig als ein Haus mit Säulen dargestellt oder es werden nur einzelne UrsacheWirkungsketten visualisiert. Eine Krankenkasse hat zudem einen BSC-Würfel für alle Mitarbeiter konzipiert, der die Vision sowie die Perspektiven und wichtigsten Ziele veranschaulicht.

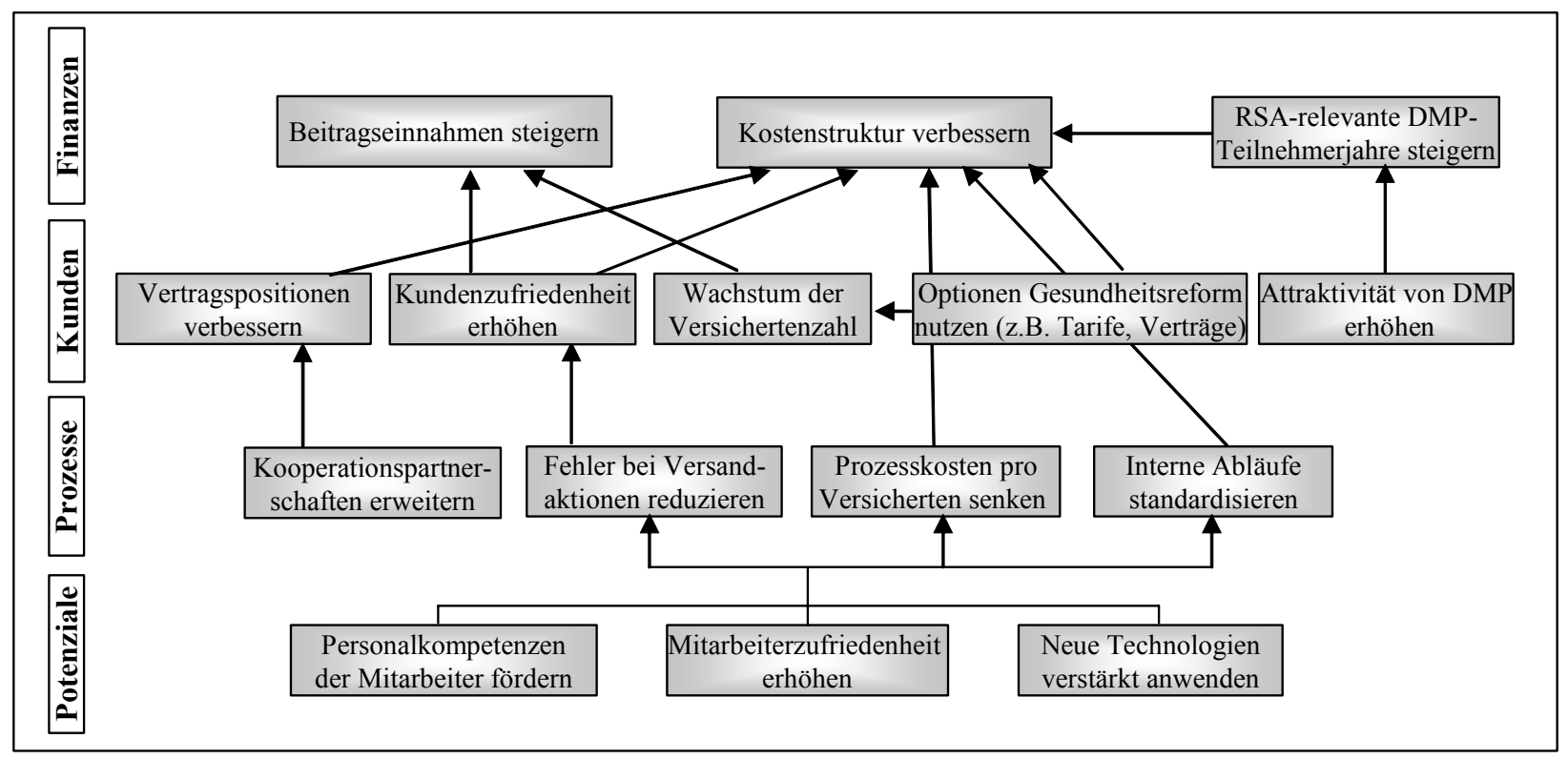

Abb. 3: Mögliche Strategielandkarte einer gesetzlichen Krankenkasse Quelle: $\quad$ Eigene Darstellung

Insgesamt zeigen die Ergebnisse der Experteninterviews vielfältige Besonderheiten der BSC-Anwendung in Krankenkassen auf. Die im Rahmen der vierten Forschungsfragestellung eher breit aufgestellte Hypothese (der Aufbau und die Ausgestaltung der BSC in gesetzlichen Krankenkassen weist spezifische Anpassungen zum BSC-Grundkonzept auf, insbesondere was die Kennzahlen betrifft, aber auch in der Perspektivenwahl und deren hierarchischer Anordnung) konnte damit im Grundsatz bestätigt werden.

\section{Zusammenfassung und Diskussion}

Die vorliegende Studie zeigt, dass bereits ein Viertel der Krankenkassen die BSC zur Steuerung ihrer Organisation einsetzen. Dabei implementieren insbesondere die größeren Krankenkassen die BSC. Dieses Ergebnis schließt, wie oben gezeigt, an weitere empirische Studien zur BSC an, die auch die Unternehmensgröße als eine Determinante für den BSC-Einsatz sehen. 
Die dargestellten Zahlen zur Verbreitung der BSC in Krankenhäusern (etwa 34 \%) und in der Industrie (etwa $50 \%$ ) verdeutlichen, dass die BSC in gesetzlichen Krankenkassen gegenüber anderen Branchen weniger häufig zum Einsatz kommt (Ahn/Reuter 2005; Bach 2006). Ferner ist aufgrund der Nicht-Teilnahme und der überdurchschnittlichen Kassengröße der teilnehmenden Krankenkassen die Quote von $26 \%$ als Obergrenze zu sehen. Vor dem Hintergrund der zumindest ehemals starken Verwaltungstätigkeit der Krankenkassen, ist ein geringerer Implementierungsgrad jedoch nicht verwunderlich. Im Gegenteil, man könnte diese Zahlen im positiven Sinne auch als Wandel zu einer stärker unternehmerischen Einstellung interpretieren, so dass hier durchaus von der Bereitschaft zu neuartigen Managementmethoden gesprochen werden kann.

Bezüglich des BSC-Einsatzes und der Kassenart konnten keine signifikanten Zusammenhänge identifiziert werden, obwohl deskriptiv deren Anwendung zwischen $20 \%$ und $80 \%$ differierte. Dies hängt vermutlich mit der teils geringen Fallzahl in der jeweiligen Kassenart zusammen.

Auch in Bezug auf die Entwicklung der Versichertenzahlen und der BSC-Anwendung wurde kein Zusammenhang festgestellt. Eine Kategorisierung der Versichertenzahlenentwicklung in eine binäre Variable (gesunken/gleichgeblieben vs. gestiegen und gesunken vs. gleichgeblieben/gestiegen) änderte an diesem Ergebnis nichts. Auch eine Betrachtung der BSC-Einsatzdauer und Versichertenentwicklung führte zu keinem Ergebnis, so dass auf Basis der vorliegenden Untersuchung keine Anhaltspunkte für einen $\mathrm{Zu}-$ sammenhang zwischen BSC-Einsatz in Krankenkassen und dem Leistungsparameter Entwicklung der Versichertenzahlen gegeben sind.

Im Einsatz von Strategien und Kennzahlensystemen präsentierten sich konsequenterweise deutliche Unterschiede bei BSC- und Nicht-BSC Krankenkassen. Der häufigere Einsatz einer Kosten- und Leistungsrechung erwies sich als Parameter, der die Wahrscheinlichkeit für den BSC-Einsatz erhöht. Die in Kapitel 2 formulierte Vermutung, dass die BSC-Anwendung Anreizsysteme mit individuellen Zielvereinbarungen fördert, konnte hingegen nicht bestätigt werden. Auch andere Studien konstatieren den BSCUnternehmen hierbei Nachholbedarf (Horváth \& Partners 2005, S. 23 f.).

Die empirische Untersuchung hat darüber hinaus gezeigt, dass trotz der spezifischen Rahmenbedingungen der Einsatz einer BSC in gesetzlichen Krankenkassen gut möglich ist. Im Hinblick auf den Einbezug neuer Perspektiven haben die Kassen einen hohen Anpassungsgrad bewiesen. Während die Krankenkassen zu „nur“ $62 \%$ die vier klassischen Perspektiven mit deren gängigen Anordnung verwenden, liegt die Quote bei den (vorwiegend) erwerbswirtschaftlichen Unternehmen aus der Übersichtsstudie von Bach bei rund $80 \%$ (Bach 2006, S. 301). Zudem wurde die in der Literatur für den öffentlichen Bereich vorgeschlagene zusätzliche Perspektive „Leistungsauftrag“ von keiner Krankenkasse aufgegriffen (Horváth \& Partners 2004, S. 442). Hingegen haben die Krankenkassen eigene Perspektiven wie etwa „Versorgung \& Leistungsangebot“ oder „Politik“ in ihren BSCs aufgenommen. Insbesondere erstere ist attraktiv für den Einbezug in die BSC einer Krankenkasse, da im Zuge jüngerer Gesetzesänderungen vielfältigere Gestaltungsmöglichkeiten bei Versorgungs- und Versicherungsformen eingeräumt wurden (Popp 
2003, S. 11). Betrachtet man jedoch die jüngsten Versuche der Krankenkassen zur politischen Einflussnahme bei der Diskussion um die Gesundheitsreform, so kann - zumindest bei größeren Krankenkassen - auch das Hinzuziehen einer Politikperspektive eine sinnvolle Ergänzung des BSC-Grundmodells sein.

Bezüglich des Portfolios an Kennzahlen haben die Krankenkassen ebenfalls einen hohen Anpassungsgrad bewiesen. Klassische Kennzahlen aus dem erwerbswirtschaftlichen Bereich wie Jahresüberschüsse und Eigenkapitalrentabilität können aufgrund des oben skizzierten öffentlichen Auftrags nicht als finanzielle Ziele für Krankenkassen dienen (Schlösser/Schreyögg 2005, S. 326). Wie die empirische Untersuchung zeigte, haben die Krankenkassen spezifische Zielsetzungen etwa im Bereich der Leistungen, Verwaltungskosten und Rücklagen in ihre Finanzperspektiven aufgenommen. Die per Gesetz erweiterten Gestaltungsspielräume zum selektiven Kontrahieren spiegeln sich zudem in den neu kreierten Perspektiven Versorgung \& Leistungsangebot und Produkte der Krankenkassen wider.

Wie einleitend erwähnt, ist die BSC aufgrund der Integrierbarkeit nicht-quantitativer Erfolgsgrößen besonders interessant für die Krankenkassen. Die weniger dominante Stellung der Finanzperspektive an der Spitze der BSC unterstreicht dies. Mehr als die Hälfte der Krankenkassen verzichtet auf eine hierarchische Anordnung der Perspektiven. Dies erklärt unter anderem, dass nur ein Drittel der Krankenkassen sich umfassend mit Ursache-Wirkungsbeziehungen über die verschiedenen Perspektiven hinweg befasst. Weitere Studien zeigen, dass von den befragten Unternehmen rund $60 \%$ mit UrsacheWirkungsketten arbeiten, also rund $25 \%$ häufiger als dies in Krankenkassen der Fall ist (Bach 2006, S. 301). Eines der zentralen BSC-Elemente wird daher von den meisten Krankenkassen vernachlässigt. ${ }^{18}$ Hinzu kommt, dass einige der Krankenkassen die BSC nur auf oberster Hierarchieebene implementiert haben und keine Kommunikation der BSC-Idee an den weiteren Mitarbeiterstab erfolgte. Ein Einsatz von BSCs für jeden Mitarbeiter ist aufgrund der Komplexität zwar schwer machbar (Ruhtz 2001, S. 10), dennoch widerspricht die teils geringe Implementierungstiefe dem BSC-Gedanken, nach dem eine möglichst tiefe Übersetzung der Strategie in operatives Handeln erfolgen soll. Zudem schmälert eine geringe Implementierungstiefe die Effektivität der BSC (Ahn/Reuter 2005, S. 616). Mit ihrer Implementierungstiefe liegen die Krankenkassen zwar leicht über denen aus anderen Branchen (Bach 2006, S. 301), gleichwohl ist auch hier Handlungsbedarf zu konstatieren.

Die in der Studie erzielten Rücklaufquote von $34 \%$ kann im Vergleich zu anderen Studien als „gut" bewertet werden (Haenecke 2001, S. 155). Die Überrepräsentativität der großen Krankenkassen in der vorliegenden Studie führt hingegen zu Einschränkungen, was beispielsweise, wie oben bereits angesprochen, die BSC-Quote als Obergrenze erscheinen lässt. Zudem basieren die Ergebnisse der Expertenbefragung auf „nur“ 13 Experteninterviews. Diesbezügliche quantitative Aussagen sind daher vorsichtig zu betrachten.

18 Die Vernachlässigung der Ursache-Wirkungsbeziehungen wird auch von anderen Autoren bemängelt (Speckbacher/Bischof 2000, S. 806 ff.). 


\section{Managementimplikationen}

Auf der Basis der konstatierten Defizite der Untersuchung werden im Folgenden Vorschläge für einen verbesserten Einsatz der BSC generiert. Diese sind zum einen an gesetzliche Krankenkassen gerichtet, die bereits mit der BSC arbeiten, zum anderen an Krankenkassen und weitere Organisationen, die den BSC-Einsatz planen:

Die Krankenkassen sollten eingehend prüfen, welche (weiteren) Perspektiven in ihrer Organisation am besten geeignet sind. Vor dem Hintergrund der zunehmenden Gestaltungsmöglichkeiten im Leistungsbereich wie auch der Unwägbarkeiten von politischer Seite empfiehlt sich eine Aufnahme der Perspektiven „Versorgung und Leistungsangebot" sowie „Politik“. Bei der Perspektivenwahl sollte Raum für Abweichungen gegeben sein. So könnten kunden- oder politikferne Arbeitsbereiche auf die entsprechenden Perspektiven verzichten, womit eine Komplexitätsreduktion verbunden wäre.

Die BSC-Perspektiven sollten von den Krankenkassen in einer klaren Hierarchie angeordnet sein. Nur so lassen sich konsequent Ursache-Wirkungsbeziehungen erstellen. Die Diskussion der Ursache-Wirkungsbeziehungen ist kein Selbstzweck, sondern dient dem Begreifen des gesamten Geschäftsverständnisses. Die Betrachtung einzelner Zusammenhänge ist sinnvoll, vernachlässigt aber den ganzheitlichen Ansatz der BSC. Eine Visualisierung der wichtigsten Beziehungen in einer Strategielandkarte erleichtert zudem deren Kommunikation. Durch den Einbezug einer Ampelfunktion, eines Vorzeichens oder verschiedener Pfeilstärken können Bedeutungen hervorgehoben werden.

Grundsätzlich empfiehlt sich eine tiefgehende Implementierung der BSC in den Krankenkassen, um damit die Strategieumsetzung sicherzustellen - wobei Aufwand und Ertrag zu prüfen sind. Nur so kann gewährleistet werden, dass die Strategie bei den Mitarbeitern ankommt und diese ihren Beitrag zum Unternehmenserfolg erkennen. Ist dies nicht möglich, sollte zumindest eine breite Kommunikation der Strategie und der BSC erfolgen. Dabei empfiehlt sich eine am Mitarbeiterstab ausgerichtete Anpassung der Begrifflichkeiten (die OÖGKK nennt die BSC beispielsweise „Erfolgsplan®”, vgl. Wesenauer 2006, S. 31).

Anreizsysteme mit individuellen Zielvereinbarungen fördern prinzipiell die Umsetzung der Zielsetzungen im Unternehmen, wobei den Krankenkassen eine konsequentere Verknüpfung mit der BSC nahegelegt wird. Gleichzeitig sei auf die Gefahren von anreizorientierten Vergütungssystemen wie etwa der Subjektivität und Manipulation nicht finanzieller Kennzahlen oder der Zielgewichtung hingewiesen (Pfaff et al. 2000, S. 45 ff.). Eine Möglichkeit, Mitarbeiter breit am Erfolg teilhaben zu lassen, wäre eine auf der BSC basierende Erfolgsprämie. 


\section{Schlussfolgerungen und Forschungsbedarf}

Die Umbruchsituation in der GKV zwingt die gesetzlichen Krankenkassen, neben den operativen Anpassungsmechanismen, strategisches Handeln stärker in den Mittelpunkt zu rücken. Die vorliegende Studie zeigt, dass bereits ein Viertel der befragten Krankenkassen dieser Herausforderung mit der BSC begegnen. Die Krankenkassen passen den Aufbau der BSC und deren Inhalte an die spezifischen Rahmenbedingungen an und beweisen damit teilweise ein beträchtliches innovatives Potenzial. Die hohe Erfolgsquote bei der Einführung und deren positive Bewertung seitens der Krankenkassen zeigt ferner die Eignung der BSC als Steuerungsinstrument für gesetzliche Krankenkassen, nicht zuletzt wegen des Integrationspotenzials qualitativer Erfolgsgrößen. Daneben offenbaren sich aber auch Defizite in der Umsetzung einzelner BSC-Aspekte. Zudem konnte kein kausaler Zusammenhang zwischen BSC-Einsatz und Performance der Krankenkassen (hier: Die Entwicklung der Versichertenzahlen) nachgewiesen werden.

Die Erfahrungsberichte zur BSC im deutschen Sprachraum kommen fast ausschließlich aus dem privatwirtschaftlichen Sektor. Der deutschsprachige Non-Profit-Bereich hat die Nutzenpotenziale noch nicht in vollem Umfang erkannt (Horváth/Gaiser 2000, S. 34). Der vorliegende Beitrag kann daher als Anwendungsbeispiel für Krankenversicherungen, welche die BSC nutzen oder einführen wollen, und auch für andere Organisationen und Unternehmen aus dem Non-Profit-Bereich dienen.

Weiterer Forschungsbedarf ergibt sich sowohl für detailspezifische Analysen als auch für kausalanalytische Untersuchungen, die den Erfolg oder Misserfolg einer BSC messen. Ein von den Krankenkassen genanntes und in der Literatur häufig beschriebenes Problem ist die unzureichende Messbarkeit nicht-finanzieller Kennzahlen (Hankeln 2004, S. 99). Gerade für gesetzliche Krankenkassen, bei denen die Finanzperspektive eine weniger dominante Rolle spielt als in erwerbswirtschaftlichen Unternehmen, wären geeignete Ansätze für die Messung dieser ,weichen“ Faktoren bedeutsam.

\section{Abstract}

Oliver Gapp; The Balanced Scorecard in the statutory health insurance: an empirical study of the implementation and design

Balanced Scorecard; competition; controlling; empirical study; healthcare system; management; sickness fund; statutory health insurance

The usefulness of the Balanced Scorecard (BSC) as a management tool for German sickness funds has been discussed for a few years. However empirical studies are still missing. The aim of this study is to investigate empirically the level of awareness and implementation as well as the design of the BSC in sickness funds. Furthermore characteristics of BSC-funds and the application of (BSC-related) management instruments will be ex- 
amined. In a comprehensive survey 247 sickness funds were contacted with a questionnaire and structured interviews were preformed. The results show amongst others, that with appropriate adjustments the BSC seems to be a suitable management tool for sickness funds, in particular because of its integration of qualitative measures. However, the diffusion of the BSC in sickness funds is behind other branches.

\section{Literaturverzeichnis}

Ahn, Heinz (2003), Ableitung unternehmensspezifischer Balanced Scorecards: Anspruch, Realität und Verbesserungsansatz, in: Zeitschrift für Planung und Unternehmenssteuerung, 14. Jg., S. 127-148.

Ahn, Heinz und Stefan Reuter (2005), Balanced Scorecard fasst in Krankenhäusern nur langsam Fuß, in: führen und wirtschaften im Krankenhaus (f\&w), 22. Jg., Heft 6, S. 614-616.

Bach, Norbert (2006), Analyse der empirischen Balanced Scorecardforschung im deutschsprachigen Raum, in: Zeitschrift für Controlling und Management, 50. Jg., Heft 5, S. 298-304.

Backhaus, Klaus, Wulff Plinke, Bernd Erichson und Rolf Weiber (2006), Multivariate Analysemethoden. Eine anwendungsorientierte Einführung, 11. Aufl., Berlin u. a.

Blaudszun, Markus und Renate Pielniok (2002), Software unterstützt Balanced Scorecard-Prozess, in: Versicherungsbetriebe, o. Jg., Heft 6, S. 38-40.

Böhm, Karin, Michael Cordes, Anja Afentakis, Michael Müller und Manuela Nöthen (2006), Gesundheit - Ausgaben, Krankheitskosten und Personal 2004. Wiesbaden.

Borges, Peter und Ralf Schmidt (2002), Die Balanced Scorecard als Steuerungsinstrument im Krankenhaus, in: Betriebswirtschaftliche Forschung und Praxis (BFuP), o. Jg., Heft 2, S. 101-117.

Bundesministerium für Gesundheit (2006), Statistik über Versicherte, gegliedert nach Status, Alter, Wohnort, Kassenart 2005, www.bgms.bund.de (Zugriff: 16.01.2006).

Cassel, Dieter (1993), Anspruch und Wirklichkeit - Zur Organisationsreform der GKV, in: Soziale Sicherheit, 42. Jg., Heft 2, S. 18-24.

Chow, Chee W., Denise Ganulin, Kamal Haddad und James Williamson (1998), The balanced scorecard: a potent tool for energizing and focusing healthcare organization management, in: Journal of Healthcare Management, 43. Jg., Heft 3, S. 263-280.

Demmler, Gertrud (2002), Balanced Scorecard. Von der Strategie zur Aktion ..., in: Die BKK, Heft 11, S. 485-490.

Dick, Beate, Jürgen-Christian Krieg und Wolfgang Schreiber (2002), Die „Balanced Scorecard“ als Chance für die ärztliche Klinikleitung, in: Gesundheitsökonomie \& Qualitätsmanagement, 7. Jg., Heft 3, S. 166-172.

Fink, Carmen A. und Christian Grundler (1998), Strategieimplementierung im turbulenten Umfeld, in: Controlling, 10. Jg., Heft 4, S. 226-235.

Fließ, Sabine, Britta Lasshof und Thorsten Matznick (2006), Die Balanced Scorecard für Dienstleister. Das Beispiel der Flughafen Stuttgart GmbH, in: Controlling, o. Jg., Heft 2, S. 99-106.

Bernd Gaiser und Thomas Wunder (2004), Strategy Maps und Strategieprozess: Einsatzmöglichkeiten, Nutzen, Erfahrungen, in: Controlling, o. Jg., Heft 8/9, S. 457-463.

Gapp, Oliver (2007), Umsetzungserfahrungen gesetzlicher Krankenkassen mit der Balanced Scorecard: Ein Ergebnisbericht der Gründe, Vorgehensweise und Probleme, in: Zeitschrift für die gesamte Versicherungswissenschaft, o. Jg., Heft 3, S. 281-304.

GKV (2008), Gesetzliche Krankenversicherung (GKV). Aufgaben. Abgerufen im Internet unter http://www.gkv. info/gkv/index.php?id=72 (Zugriff: 04.02.2008).

Haenecke, Henrik (2001), Krankenkassen-Marketing: eine empirische Analyse der Erfolgsfaktoren, München, u. a.

Hankeln, Christoph (2004), Entwicklung und Implementierung von Strategien im Krankenhaus mit Hilfe einer Balanced Scorecard, in: Journal für Anästhesie und Intensivbehandlung, o. Jg., Heft 1, S. 98-104.

Heinzen, Frank (2002), Strategien für ein zukunftsfähiges Versorgungssystem der gesetzlichen Krankenversicherung, E-Dissertation, Bielefeld.

Hofmann, Jörg Klaus (1999), Controlling in gesetzlichen Krankenversicherungen, Frankfurt am Main, u. a.

Hörbst, Günther (2007), Kassenchef: Ab 2009 wird es für Versicherte richtig teuer. Barmer-Chef Vöcking sagt Beitragserhöhungen durch Gesundheitsfonds voraus, Berliner Morgenpost, 6. Dezember 2007.

Horváth \& Partners (2004), Balanced Scorecard umsetzen, Stuttgart.

Horváth \& Partners (2005), Balanced-Scorecard-Studie 2005. Ergebnisbericht, Stuttgart.

Horváth, Peter (1999), Das Balanced-Scorecard-Managementsystem - Ausgangsproblem, der Lösungsansatz und die Umsetzungserfahrungen, in: Die Unternehmung, 53. Jg., Heft 5, S. 303-319.

Horváth, Peter (2001), Value Based Management and Balanced Scorecard in European Companies - An International Comparison Between Germany - Great Britain - France - Italy, Univ. Stuttgart, Betriebswirtschaftliches Inst., Lehrstuhl für Allg. Betriebswirtschaftslehre und Controlling, Stuttgart. 


\section{Oliver Gapp}

Horváth, Peter und Bernd Gaiser (2000), Implementierungserfahrungen mit der Balanced Scorecard im deutschen Sprachraum - Anstöße zur konzeptionellen Weiterentwicklung, in: Betriebswirtschaftliche Forschung und Praxis (BFuP), o. Jg., Heft 1, S. 17-35.

Inamdar, Norein und Robert S. Kaplan (2002), Applying the balanced scorecard in healthcare provider organizations, in: Journal of Healthcare Management, vol. 47, no. 3, pp. 179-195.

John, Jürgen und Reiner Leidl (2004), Zur Bedeutung der ökonomischen Evaluation bei Ein- und Ausschluss von Leistungen, in: Sozialer Fortschritt, 53. Jg., Heft 8-9, S. 219-228.

Kaplan, Robert S. (2001), Strategic Performance Measurement and Management in Nonprofit Organizations, in: Nonprofit Management \& Leadership, vol. 11, no. 3, pp. 353-370.

Kaplan, Robert S. und David P. Norton (1992), The balanced scorecard--measures that drive performance, in: Harvard Business Review, vol. 70, no. 1, pp. 71-79.

Kaplan, Robert S. und David P. Norton (1993), Putting the balanced scorecard to work, in: Harvard Business Review, vol. 71, no. 5, pp. 134-147.

Kaplan, Robert S. und David P. Norton (1997), Balanced Scorecard: Strategien erfolgreich umsetzen, Stuttgart.

Klemz, Wolfgang (2004), Ziele der Kosten- und Leistungsrechnung als Grundlage praxisorientierter Konzepte für die Betriebliche Krankenversicherung - Teil 1, in: Die BKK, o. Jg., Heft 3, S. 103-107.

Klusen, Norbert und Peter Horvath (2005), TK - Strategieorientierung einer Krankenkasse, in: Controlling, o. Jg., Heft 11, S. 685-690.

Kumar, Arun, Linet Ozdamar und Chai Peng Ng (2005), Procurement performance measurement system in the health care industry, in: International Journal of Health Care Quality Assurance Incorporating Leadership in Health Services, vol. 18, no. 2-3, pp. 152-166.

Lawrie, Gavin und Ian Cobbold (2004), Third Generation Balanced Scorecard: evolution of an effective strategic control tool, in: International Journal of Productivity and Performance Management, vol. 53, no. 7, pp. 611623 .

Marburger, Horst und Dietmar Marburger (1997), Wettbewerb unter den gesetzlichen Krankenkassen. Vertriebspolitik, Außendienst, Rechtsgrundlagen, Sankt Augustin.

Mayer, Reinhold und Helmut Ahr (2000), „Translating strategy into action“. Strategieimplementierung mit der Balanced Scorecard in Versicherungsunternehmen, in: Zeitschrift für die gesamte Versicherungswissenschaft, 98. Jg., Heft 4, S. 674-688.

Meuser, Michael und Ulrike Nagel (1991), ExperInneninterviews - vielfach erprobt, wenig bedacht. Ein Beitrag zur Methodendiskussion, in: Garz, Detlef und Klaus Kraimer: Qualitativ-empirische Sozialforschung. Methoden, Konzepte, Analysen, Opladen, S. 441-471.

Moos, Gabriele und Frank Brüggemann (2006), Informationsmanagement und Controlling in Krankenversicherungen, in: Busse, Reinhard, Jonas Schreyögg, Christian Gericke: Management im Gesundheitswesen, Heidelberg, S. 337-350.

Pfaff, Dieter, Alexis Kunz und Thomas Pfeiffer (2000), Balanced Scorecard als Bemessungsgrundlage finanzieller Anreizsysteme - Eine theorie- und empiriegeleitete Analyse der resultierenden Grundprobleme, in: Betriebswirtschaftliche Forschung und Praxis (BFuP), o. Jg., Heft 1, S. 36-55.

Popp, Ekhard (2003), Vom Einprodukt- zum Mehrproduktanbieter. Die neuen Möglichkeiten der Gesetzlichen Krankenkassen, in: Gesundheit und Sozialpolitik, 57. Jg., Heft 9-10, S. 10-17.

Puke, Stephan (1998), Controlling in Managed Care, in: Burchert, Heiko und Thomas Hering: Gesundheit und Ökonomie: interdisziplinäre Lösungsvorschläge, Baden-Baden, S. 202-216.

Radnor, Zoe und Bill Lovell (2003), Defining, justifying and implementing the Balanced Scorecard in the National Health Service, in: International Journal of Medical Marketing, vol. 3, no. 3, pp. 174-188.

Rimar, Stephen (2000), Strategic planning and the balanced scorecard for faculty practice plans, in: Acadademic Medicine, vol. 75, no. 12, pp. 1186-1188.

Romeike, Frank (2003), Balanced Scorecard in Versicherungen, Wiesbaden.

Ruhtz, Vanessa (2001), Die Balanced Scorecard im Praxistest: Wie zufrieden sind Anwender, Price Waterhouse Coopers, Deutsche Revision, Frankfurt am Main.

Sahney, Vinod K. (1998), Balanced scorecard as a framework for driving performance in managed care organizations, in: Managed Care Quarterly, vol. 6, no. 2, pp. 1-8.

Scherer, Andreas G. (2002), Besonderheiten der strategischen Steuerung in öffentlichen Institutionen und der Beitrag der Balanced Scorecard, in: Alt, Jens M. und Andreas G. Scherer: Balanced Scorecard in Verwaltung und Non-Profit-Organisationen, S. 3-22.

Schlösser, Rico und Jonas Schreyögg (2005), Die Balanced Scorecard als Kennzahlensystem für Krankenkassen, in: Zeitschrift für die gesamte Versicherungswissenschaft, 94. Jg., Heft 2, S. 323-345.

Schnell, Reiner, Paul Bernhard Hill und Elke Esser (2005), Methoden der empirischen Sozialforschung, 7. Aufl., München u. a.

Schwertner, Kerstin, Wolfgang Becker und Christoffer-Martin Seubert (2005), Strategieumsetzung mit BSCbasierten Anreizsystemen, in: Controlling, 17. Jg., Heft 1, S. 33-39.

Speckbacher, Gerhard und Jürgen Bischof (2000), Die Balanced Scorecard als innovatives Managementsystem, in: Die Betriebswirtschaft, 60. Jg., Heft 6, S. 795-810. 


\section{Balanced Scorecard in gesetzlichen Krankenkassen}

Speckbacher, Gerhard, Jürgen Bischof und Thomas Pfeiffer (2003), A descriptive analysis on the implemantation of Balanced Scorecards in German-speaking countries, in: Management Accounting Research, vol. 14, no. 4, pp. 361-387.

Weber, David (1999), Performance management--the balanced scorecard: a framework for managing complex and rapid change, in: Strategies for Healthcare Excellence, vol. 12, no. 11, pp. 1-7.

Wesenauer, Andrea (2002), Mit der Balanced Scorecard zum echten Erfolgsplan. Modernes Management in der Oberösterreichischen Gebietskrankenkasse, in: innovative VERWALTUNG, o. Jg., Heft 11, S. 13-15.

Wesenauer, Andrea (2006), Systemische Strategieentwicklung und strategisches Management auf Basis der Balanced Scorecard, in: Betriebswirtschaftliche Instrumente der Unternehmenssteuerung in der sozialen Krankenversicherung, hrsg. von Andrea Wesenauer und Werner H. Bencic, Linz, S. 17-39.

Wesenauer, Andrea und Werner H. Bencic (2006), Betriebswirtschaftliche Instrumente der Unternehmenssteuerung in der sozialen Krankenversicherung, Linz.

Zdrowomyslaw, Norbert, Veiko von Eckern und Andre Meißner (2003), Akzeptanz und Vorbereitung der Balanced Scorecard, in: Betrieb und Wirtschaft, o. Jg., Heft 9, S. 356-359. 